K. Yoshikawa

Nagoya Math. J.

Vol. 146 (1997), 83-129

\title{
DEGENERATION OF ALGEBRAIC MANIFOLDS AND THE SPECTRUM OF LAPLACIAN
}

\author{
KEN-ICHI YOSHIKAWA
}

\begin{abstract}
We shall show that the spectrum of Laplacian depends continuously on the parameter for one parameter degeneration of projective algebraic manifolds.
\end{abstract}

\section{$\S 0$. Introduction}

In [Gr1], M. Gromov studies the spectral geometry of semi-algebraic sets in the Euclidean space. He treats a family of algebraic manifolds $\left\{X_{t}\right\}$ and studies the continuity of several geometric and analytic quantities. When no degeneration of manifolds happens, most of such quantities depend continuously on the parameter. He states that the spectrum of Laplacian is continuous in the parameter unless serious degeneration happens. As for such degenerations, he mentions the case that the singular fiber has multiple components and that the dimension of the singular fiber is different from that of the general fibers (cf. [Gr1, 4C]).

The purpose of this article is to study the behavior of the spectrum of Laplacian in the case of a degenerating family of projective algebraic manifolds in a fixed complex projective space. We shall show that in the case of a one parameter families, the spectrum is continuous. Therefore discontinuity can happen only for families with many parameters. (Note that the dimension of each fiber is constant in the case of a one parameter families.)

Let $\pi: \mathfrak{X} \rightarrow \Delta(1)$ be a one parameter degenerating family of projective algebraic manifolds in $\mathbb{P}^{N}(\mathbb{C})$ over the unit disc. By this we mean that $\mathfrak{X}$ is a complex submanifold of $\mathbb{P}^{N}(\mathbb{C}) \times \Delta(1)$ and that $\pi:=\operatorname{proj}_{2} \mid \mathfrak{x}$ is a proper holomorphic surjection to $\Delta(1):=\{z \in \mathbb{C} ;|z|<1\}$. Then $X_{t}:=\pi^{-1}(t)$ is a pure dimensional projective algebraic variety. Since the discriminant locus of $\pi$ is a discrete subset in $\Delta(1)$, we may assume that $X_{t}$ is a smooth

Received May 17, 1994. 
manifold for $t \neq 0$ and that $X_{0}$ is of the form $X_{0}=\sum_{\alpha=1}^{a} m_{\alpha} Y_{\alpha}$ in the sense of divisor where $m_{\alpha} \in \mathbb{Z}_{+}$and $Y_{\alpha}$ is an irreducible variety.

Let $G$ be an arbitrary Riemannian metric of $\mathfrak{X}$. Set $g_{t}:=\left.G\right|_{X_{t}}$ for $t \neq 0$ and $g_{0}:=\left.G\right|_{X_{0}-\operatorname{Sing}\left(X_{0}\right)}$. Let $\Delta_{t}$ be the Laplacian of $\left(X_{t}, g_{t}\right)$, and $\sigma\left(\Delta_{t}\right)$ the spectrum of $\Delta_{t}$. When $t=0$, consider the Dirichlet Laplacian of $X_{0, \text { reg }}:=$ $\sum_{\alpha} m_{\alpha} Y_{\alpha, \text { reg }}$; i.e., $\Delta_{0}:=\oplus_{\alpha} m_{\alpha} \Delta_{\alpha}$ where $\Delta_{\alpha}$ is the Laplacian whose domain is given by $W_{0}^{1,2}\left(Y_{\alpha, \text { reg }},\left.g_{0}\right|_{Y_{\alpha}}\right)$ where $Y_{\alpha, \text { reg }}:=Y_{\alpha}-\operatorname{Sing}\left(Y_{\alpha}\right)$, and $m_{\alpha} \Delta_{\alpha}$ is the $m_{\alpha}$-th copy of $\Delta_{\alpha}$. By the definition, $\sigma\left(\Delta_{0}\right)=\bigcup_{\alpha=1}^{a} m_{\alpha} \sigma\left(\Delta_{\alpha}\right)$. It is known that $\sigma\left(\Delta_{0}\right)$ consists of discrete eigenvalues (cf. [L-T, §5]). Naively, it seems that $\sigma\left(\Delta_{t}\right)$ converges to $\sigma\left(\Delta_{0}\right)$, since $X_{t}$ converges to $X_{0}$ in the sense of current on $\mathbb{P}^{N}(\mathbb{C})$ (cf. [F, Proposition 2.3]). But this does not hold in general, unless $X_{0}$ is a reduced divisor; i.e., $m_{\alpha}=1$ for all $\alpha$. We shall show that $\sigma\left(\Delta_{t}\right)$ converges to the spectrum of a certain branched covering space of $X_{0}$. This covering space is described as follows (cf. [Cl]).

Let $(\Delta(1), s)$ be the unit disc in the $s$-plane, i.e., the complex plane whose coordinate is given by $s$, and let $F$ be a holomorphic function defined by $F(s):=s^{m}$ where $m:=\prod_{\alpha} m_{\alpha}$. By this maps, we obtain the fiber product

$$
F^{-1} \mathfrak{X}:=\left\{(x, s) \in \mathfrak{X} \times \Delta(1) ; \pi(x)=s^{m}\right\} \subset \mathfrak{X} \times(\Delta(1), s) .
$$

Let $\Pi: F^{-1} \mathfrak{X} \rightarrow(\Delta(1), s)$ be the natural projection induced by that of $\mathfrak{X}$. Then its fiber is given by $\Pi^{-1}(t)=X_{t^{m}}$. In particular, $\Pi^{-1}(0)=X_{0}$.

Let $\iota: \widehat{F^{-1}} \mathfrak{X} \rightarrow F^{-1} \mathfrak{X}$ be the normalization of $F^{-1} \mathfrak{X}$. Finally, set $Z:=\iota^{-1}\left(X_{0}\right)=\iota^{-1}\left(\Pi^{-1}(0)\right)$ and $g_{Z}:=\iota^{*} g_{0}$.

Main Theorem. Let $\Delta_{Z}$ be the Dirichlet Laplacian of $\left(Z_{\mathrm{reg}}, g_{Z}\right)$, and $\sigma\left(\Delta_{Z}\right)$ its spectrum. Then,

$$
\lim _{t \rightarrow 0} \sigma\left(\Delta_{t}\right)=\sigma\left(\Delta_{Z}\right)
$$

In particular, the $k$-th eigenvalue of the Laplacian is a continuous function on $\Delta(1)$.

In $[\mathrm{Fk}]$, continuity of the spectrum is studied relative to the measured Hausdorff topology, and in $[K-K 1,2]$, relative to the spectral distance of Riemannian manifolds. In [J-W], the same problem is studied for a degenerating family of surfaces with respect to several metrics, and in $[Y 1,2]$, 
for a conic degenerating family of Riemannian manifolds and degenerating family of algebraic curves. Similar to these articles, the proof of Main Theorem is based on the min-max principle developed in $[\mathrm{C}],[\mathrm{C}-\mathrm{F}]$ and $[\mathrm{J}-\mathrm{W}]$. To apply these arguments to our situation, uniformity of the Sobolev constant for algebraic varieties in a fixed projective space is crucial, which is due to Li-Tian [L-T]. In the same article, it is also proved that $d_{\max }=d_{\min }$ on the space of functions for singular algebraic varieties ([L-T, Theorem 4.1]). In view of their argument, it is equivalent to the existence of a sequence of cut-off functions approximating the constant function in the $W^{1,2}$-norm. It seems that their construction of such functions contains a gap, because it is not clear whether various formulae and estimates in $[\mathrm{G}]$ hold for singular subvarieties in a singular variety. In this article, we shall give a rigorous proof of their theorem.

In view of Theorem 5.1, we can prove that the spectrum of Laplacian is a continuous function of a certain family of Einstein manifolds (cf. [B-K-N], $[\mathrm{N}])$. We conjecture that the same is true for the case treated in $[\mathrm{A}]$, and for the case of a degenerating family of minimal submanifolds of $S^{N}$, since the Sobolev inequality is uniform in the parameter in both cases (cf. [C-L-Y]).

This article is arranged as follows. In $\S 1$, we give a detailed definition of $Z$. In $\S 2$, we recall the results on the Sobolev inequality and the upper bound of the heat kernel. In $\S 3$, we discuss the existence of a certain sequence of cut-off functions on arbitrary irreducible algebraic varieties. In $\S 4$, we recall the result of $\mathrm{Li}$ and Tian (cf. [L-T]) which is the main tool of this paper. In $\S 5$, we prove an abstract version of Main Theorem (Theorem 5.1). Here, the Sobolev inequality with uniform Sobolev constant is used essentially in stead of the curvature bound. For simplicity, we prove Theorem 5.1 when $\operatorname{dim} M>2$. When $\operatorname{dim} M=2$, we can prove the theorem in the same way, if we use the Sobolev inequality obtained in Corollary 4.2. In $\S 6$, we prove Main Theorem. In Appendix, we prove some results concerning dimension and degree of algebraic varieties for the convenience of the reader. The proof given there is due to Y. Namikawa and M. Hashimoto.

Acknowledgments. The author wishes to express his thanks to the referee who pointed out several mistakes of the earlier version of this article and gave a useful suggestions for the contents of $\S 5$, and to Professor M. Hashimoto for kindly teaching him a detailed proof of Proposition A.1 
and A.2. He also wishes to express his thanks to Professors R. Kobayashi, S. Mukai, Y. Namikawa, T. Ohsawa and H. Umemura for helpful conversations and comments.

\section{$\S 1$. Description of $Z$}

Let $\pi: \mathfrak{X} \rightarrow \Delta(1)$ be the same as in the introduction. Set $X_{t}:=\pi^{-1}(t)$. When $t=0$, we have

$$
X_{0}=\sum_{\alpha=1}^{a} m_{\alpha} Y_{\alpha}
$$

as a divisor where $m_{\alpha} \in \mathbb{Z}_{+}$and $Y_{\alpha}$ is an irreducible algebraic variety. Let $\left(\Delta(1), t_{\alpha}\right)$ and $(\Delta(1), s)$ be the unit disc in the $t_{\alpha}$-plane and the $s$-plane respectively. Let $f_{\alpha}$ and $F$ be holomorphic functions defined by

$$
f_{\alpha}\left(t_{\alpha}\right):=t_{\alpha}^{m_{\alpha}}, \quad F(s):=s^{m}
$$

where $m:=\prod_{\alpha} m_{\alpha}$, by which we obtain the fiber product

$$
f_{\alpha}^{-1} \mathfrak{X}:=\left\{\left(x, t_{\alpha}\right) \in \mathfrak{X} \times \Delta(1) ; \pi(x)=t_{\alpha}^{m_{\alpha}}\right\} \subset \mathfrak{X} \times\left(\Delta(1), t_{\alpha}\right)
$$

and

$$
F^{-1} \mathfrak{X}:=\left\{(x, s) \in \mathfrak{X} \times \Delta(1) ; \pi(x)=s^{m}\right\} \subset \mathfrak{X} \times(\Delta(1), s) .
$$

Let $\pi_{\alpha}: f_{\alpha}^{-1} \mathfrak{X} \rightarrow\left(\Delta(1), t_{\alpha}\right)$ and $\Pi: F^{-1} \mathfrak{X} \rightarrow(\Delta(1), s)$ be the natural projections induced by that of $\mathfrak{X}$ whose fibers are given by $\pi_{\alpha}^{-1}(t)=X_{t^{m_{\alpha}}}$ and $\Pi^{-1}(t)=X_{t^{m}}$. In particular, $\pi_{\alpha}^{-1}(0)=\Pi^{-1}(0)=X_{0}$.

We denote the normalization of $f_{\alpha}^{-1} \mathfrak{X}$ and $F^{-1} \mathfrak{X}$ by

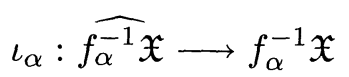

and

$$
\iota: \widehat{F^{-1} \mathfrak{X}} \longrightarrow F^{-1} \mathfrak{X}
$$

Then, regarding $X_{0}=\pi_{\alpha}^{-1}(0)=\Pi^{-1}(0) \subset F^{-1} \mathfrak{X}$ and $Y_{\alpha} \subset X_{0}$, define $Z$, $Z_{\alpha}$ and $W_{\alpha}$ by

$$
Z:=\iota^{-1}\left(X_{0}\right), \quad Z_{\alpha}:=\iota^{-1}\left(Y_{\alpha}\right), \quad W_{\alpha}:=\iota_{\alpha}^{-1}\left(Y_{\alpha}\right) .
$$


Since $(\Delta(1), s)$ is a (branched) covering of $\left(\Delta(1), t_{\alpha}\right)$, regarding $t_{\alpha}^{m_{\alpha}}=s^{\frac{m}{m_{\alpha}}}$, we obtain the natural covering map

$$
g_{\alpha}: F^{-1} \mathfrak{X} \ni(x, s) \longrightarrow\left(x, s^{\frac{m}{m_{\alpha}}}\right) \in f_{\alpha}^{-1} \mathfrak{X} .
$$

Clearly $g_{\alpha}$ is a branched $m / m_{\alpha}$-sheeted covering (cf. [G-R, pp. 135]). Since $g_{\alpha}$ is a finite surjective holomorphic map, it naturally induces a finite sur-

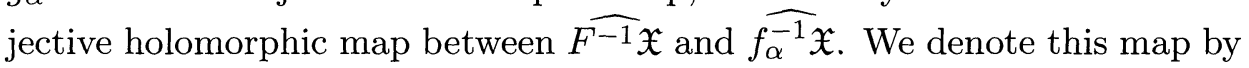
$\hat{g}_{\alpha}$ :

$$
\hat{g}_{\alpha}: \widehat{F^{-1} \mathfrak{X}} \longrightarrow \widehat{f_{\alpha}^{-1} \mathfrak{X}} .
$$

By the definition, it is clear that $\hat{g}_{\alpha}$ is a branched $m / m_{\alpha}$-sheeted covering. Restricting $\hat{g}_{\alpha}$ to the fiber at zero, we get the finite surjection

$$
\hat{g}_{\alpha}: Z_{\alpha} \longrightarrow W_{\alpha}
$$

Set $\mathfrak{X}_{\alpha}:=\widehat{f_{\alpha}^{-1} \mathfrak{X}}, p_{\alpha}:=\pi_{\alpha} \circ \iota_{\alpha}$ and define a family

$$
p_{\alpha}: \mathfrak{X}_{\alpha} \longrightarrow\left(\Delta(1), t_{\alpha}\right)
$$

whose fiber at $t \neq 0$ is the same one as that of $f_{\alpha}^{-1} \mathfrak{X}$, and therefore is $X_{t^{m_{\alpha}}}$, since $f_{\alpha}^{-1} \mathfrak{X}$ is smooth outside $X_{0}=\pi_{\alpha}^{-1}(0)$. Set

$$
\begin{aligned}
& D_{\alpha}:=\operatorname{Sing}\left(Y_{\alpha}\right) \cup \bigcup_{\beta \neq \alpha} Y_{\alpha} \cap Y_{\beta}, \\
& \Sigma_{\alpha}:=\iota_{\alpha}^{-1}\left(D_{\alpha}\right), \quad S_{\alpha}:=\hat{g}_{\alpha}^{-1}\left(\Sigma_{\alpha}\right), \quad S:=\cup_{\alpha} S_{\alpha} .
\end{aligned}
$$

Proposition 1.1. (1) $\iota: Z_{\alpha} \rightarrow Y_{\alpha}$ and $\iota_{\alpha}: W_{\alpha} \rightarrow Y_{\alpha}$ are $m_{\alpha}$-sheeted analytic covering, and $\hat{g}_{\alpha}: Z_{\alpha} \rightarrow W_{\alpha}$ is a surjective one sheeted analytic covering. In fact, $\iota: Z_{\alpha}-S_{\alpha} \rightarrow Y_{\alpha}-D_{\alpha}$ and $\iota_{\alpha}: W_{\alpha}-\Sigma_{\alpha} \rightarrow Y_{\alpha}-D_{\alpha}$ are unramified $m_{\alpha}$-sheeted covering, and $\hat{g}_{\alpha}: Z_{\alpha}-S_{\alpha} \rightarrow W_{\alpha}-\Sigma_{\alpha}$ is an isomorphism.

(2) $\operatorname{Sing}\left(W_{\alpha}\right) \subset \Sigma_{\alpha}$ and $\operatorname{Sing}\left(Z_{\alpha}\right) \subset S_{\alpha}$.

(3) $W_{\alpha}-\Sigma_{\alpha} \subset \mathfrak{X}_{\alpha, \text { reg }}$ and $p_{\alpha}$ is of maximal rank on $W_{\alpha}-\Sigma_{\alpha}$.

Proof. Let $x$ be an arbitrary point in $Y_{\alpha}-D_{\alpha}$. There is a coordinate neighborhood $\left(U_{x},\left(z_{0}, \cdots, z_{n}\right)\right)$ around $x$ in $\mathfrak{X}$ such that $x=(0, \cdots, 0)$ and for $y=\left(z_{0}, \cdots, z_{n}\right) \in U_{x} \cong \Delta(1)^{n+1}$,

$$
\pi(y)=z_{0}^{m_{\alpha}},
$$


since $x$ is a smooth point of $Y_{\alpha}$ and the multiplicity of $Y_{\alpha}$ is $m_{\alpha}$. Put

$$
\begin{aligned}
& f_{\alpha}^{-1} U_{x}:=\left\{\left(y, t_{\alpha}\right) \in f_{\alpha}^{-1} \mathfrak{X} ; y \in U_{x}\right\} \\
& F^{-1} U_{x}:=\left\{(y, s) \in F^{-1} \mathfrak{X} ; y \in U_{x}\right\} .
\end{aligned}
$$

Using above local coordinates, we can write

$$
\begin{aligned}
f_{\alpha}^{-1} U_{x} & =\left\{\left(z, t_{\alpha}\right) \in \Delta(1)^{n+1} \times \Delta(1) ; z_{0}^{m_{\alpha}}=t_{\alpha}^{m_{\alpha}}\right\}, \\
& =\left\{\left(z, t_{\alpha}\right) ; \prod_{k=1}^{m_{\alpha}}\left(z_{0}-\zeta_{m_{\alpha}}^{k} t_{\alpha}\right)=0\right\} \\
F^{-1} U_{x} & =\left\{(z, s) \in \Delta(1)^{n+1} \times \Delta(1) ; z_{0}^{m_{\alpha}}=s^{m}\right\} \\
& =\left\{(z, s) ; \prod_{k=1}^{m_{\alpha}}\left(z_{0}-\zeta_{m_{\alpha}}^{k} s^{\frac{m}{m_{\alpha}}}\right)=0\right\}
\end{aligned}
$$

where $\zeta_{m_{\alpha}}=\exp \left(2 \pi \sqrt{-1} / m_{\alpha}\right)$. From the definition of normalization, it follows that

$$
\sharp \iota_{\alpha}^{-1}(x)=\sharp \iota^{-1}(x)=m_{\alpha}
$$

and

$$
\begin{gathered}
\iota_{\alpha}^{-1} f_{\alpha}^{-1} U_{x}=\cup_{k=1}^{m_{\alpha}} V_{x, k}, \quad V_{x, k} \cap V_{x, l}=\emptyset \quad(k \neq l) \\
\iota^{-1} F^{-1} U_{x}=\cup_{k=1}^{m_{\alpha}} O_{x, k}, \quad O_{x, k} \cap O_{x, l}=\emptyset \quad(k \neq l)
\end{gathered}
$$

where

$$
\begin{gathered}
\iota_{\alpha}: V_{x, k} \longrightarrow\left\{\left(z, t_{\alpha}\right) ; z_{0}-\zeta_{m_{\alpha}}^{k} t_{m_{\alpha}}=0\right\}, \\
\iota: O_{x, k} \longrightarrow\left\{(z, s) ; z_{0}-\zeta_{m_{\alpha}}^{k} s^{\frac{m}{m_{\alpha}}}=0\right\}
\end{gathered}
$$

are isomorphisms. Set $x_{\alpha, k}:=\iota_{\alpha}^{-1}(x) \cap V_{x, k}$ and $x_{k}:=\iota^{-1}(x) \cap O_{x, k}$. Then $V_{x, k}$ (resp. $O_{x, k}$ ) is a coordinate neighborhood of $\widehat{f_{\alpha}^{-1} \mathfrak{X}}$ (resp. $\widehat{F^{-1} \mathfrak{X}}$ ) around $x_{\alpha, k}$ (resp. $x_{x}$ ). Therefore $V_{x, k} \cap W_{\alpha}$ (resp. $\left.O_{x, k} \cap Z_{\alpha}\right)$ is a coordinate neighborhood of $W_{\alpha}\left(\operatorname{resp} . Z_{\alpha}\right)$ around $x_{\alpha, k}\left(\right.$ resp. $\left.x_{x}\right)$ :

$(1.23) \iota_{\alpha}: V_{x, k} \cap W_{\alpha} \rightarrow\left\{\left(z, t_{\alpha}\right) \in \Delta(1)^{n+1} \times \Delta(1) ; z_{0}=t_{m_{\alpha}}=0\right\} \cong \Delta(1)^{n}$, 


$$
\iota: O_{x, k} \cap Z_{\alpha} \rightarrow\left\{(z, s) \in \Delta(1)^{n+1} \times \Delta(1) ; z_{0}=s=0\right\} \cong \Delta(1)^{n},
$$

which implies that $W_{\alpha}-\Sigma_{\alpha}$ and $Z_{\alpha}-S_{\alpha}$ consist of smooth points of $W_{\alpha}$ and $Z_{\alpha}$ respectively, since $x$ is an arbitrary point of $Y_{\alpha}-D_{\alpha}$. This proves (2).

By (1.19), (1.20), (1.23) and (1.24), $\iota_{\alpha}$ (resp. $\left.\iota\right)$ is a $m_{\alpha}$-sheeted covering map between $W_{\alpha}-\Sigma_{\alpha}$ and $Y_{\alpha}-D_{\alpha}$ (resp. $Z_{\alpha}-S_{\alpha}$ and $\left.Y_{\alpha}-D_{\alpha}\right)$. This proves (1) for $\iota_{\alpha}$ and $\iota$. Since $g_{\alpha}(z, s)=\left(z, s^{\frac{m}{m_{\alpha}}}\right)$ in the above coordinates, $g_{\alpha}$ : $\iota\left(O_{x, k}\right) \rightarrow \iota_{\alpha}\left(V_{x, k}\right)$ is an isomorphism. Therefore we obtain an isomorphism $\hat{g}_{\alpha}: O_{x, k} \rightarrow V_{x, k}$. As $\hat{g}_{\alpha}$ is globally defined on $Z_{\alpha}$, it must be an unramified covering resiricted to $Z_{\alpha}-S_{\alpha}$. Since $g_{\alpha} \circ \iota=\iota_{\alpha} \circ \hat{g}_{\alpha}$ and $g_{\alpha}$ is the identity map on $Y_{\alpha}, \hat{g}_{\alpha}$ must be an isomorphism between $Z_{\alpha}-S_{\alpha}$ and $W_{\alpha}-\Sigma_{\alpha}$, comparing the mapping degree. This completes the proof of (1).

By (1.21), there exists a coordinate neighborhood $\left(V_{x, k},\left(\xi_{0}, \cdots, \xi_{n}\right)\right)$ around $x_{k}$ such that $x_{k}=(0, \cdots, 0)$ and for $\xi=\left(\xi_{0}, \cdots, \xi_{n}\right) \in V_{x, k}$,

$$
\xi_{k}:=\iota_{\alpha}^{*} z_{k}, \quad p_{\alpha}(\xi)=\zeta_{m_{\alpha}}^{-k} \xi_{0} .
$$

This shows that $p_{\alpha}$ is of maximal rank at $x_{k}$ and that $p_{\alpha}$ is of maximal rank on $W_{\alpha}-\Sigma_{\alpha}$. This proves (3).

Define the $\epsilon$-tubular neighborhood of $\Sigma_{\alpha}$ and $S_{\alpha}$ as follows. By the definition, $f_{\alpha}^{-1} \mathfrak{X}$ is a subset of $A:=\mathbb{P}^{N}(\mathbb{C}) \times(\Delta(1), t) \times\left(\Delta(1), t_{\alpha}\right)$. Let $G_{A}$ be a Riemannian metric of $A$ defined by

$$
G_{A}:=g_{\mathbb{P}^{N}}+|d t|^{2}+\left|d t_{\alpha}\right|^{2}
$$

where $g_{\mathbb{P}^{N}}$ is the Fubini-Study metric of $\mathbb{P}^{N}(\mathbb{C})$. Let $d_{A}(\cdot, \cdot)$ be the distance of $A$ induced by $G_{A}$. Then, define a function of $f_{\alpha}^{-1} \mathfrak{X} \times f_{\alpha}^{-1} \mathfrak{X}$ by

$$
d_{\alpha}(x, y):=d_{A}(i(x), i(y))
$$

where $i: f_{\alpha}^{-1} \mathfrak{X} \rightarrow A$ is the natural inclusion. By the definition, it is clear that for any $x, y \in \pi_{\alpha}^{-1}(t)$,

$$
d_{\alpha}(x, y)=d_{\mathbb{P}^{N}}\left(j_{t}(x), j_{t}(y)\right)
$$

where $j_{t}: \pi_{\alpha}^{-1}(t) \hookrightarrow \mathbb{P}^{N}(\mathbb{C})$ is the natural inclusion. 
Regarding $\pi_{\alpha}^{-1}(0)=X_{0}$, define the $\epsilon$-neighborhood of $D_{\alpha}$ by

$$
D_{\alpha, \epsilon}:=\left\{x \in f_{\alpha}^{-1} \mathfrak{X} ; d_{\alpha}\left(x, D_{\alpha}\right)<\epsilon\right\},
$$

and the $\epsilon$-neighborhood of $\Sigma_{\alpha}, S_{\alpha}$ and $S$ by

$$
\Sigma_{\alpha, \epsilon}:=\iota_{\alpha}^{-1}\left(D_{\alpha, \epsilon}\right), \quad S_{\alpha, \epsilon}:=\hat{g}_{\alpha}^{-1}\left(\Sigma_{\alpha, \epsilon}\right), \quad S_{\epsilon}:=\cup S_{\alpha, \epsilon} .
$$

As $Y_{\alpha}-D_{\alpha, \epsilon}=\left\{y \in Y_{\alpha} ; d_{\mathbb{P}^{N}}\left(y \cdot D_{\alpha}\right) \geq \epsilon\right\}$ is compact, so are $W_{\alpha}-\Sigma_{\alpha, \epsilon}$, $Z_{\alpha}-S_{\alpha, \epsilon}$ and $Z-S_{\epsilon}$ by Proposition 1.1.

Let $G$ be the same Riemannian metric of $\mathfrak{X}$ as in introduction. Let $G_{\alpha}$ (resp. $H_{\alpha}$ ) be a Riemannian metric of $f_{\alpha}^{-1} \mathfrak{X}\left(\right.$ resp. $\left.\mathfrak{X}_{\alpha}\right)$, defined by

$$
G_{\alpha}:=G+\left.\left|d t_{\alpha}\right|^{2}\right|_{f_{\alpha}^{-1} \mathfrak{X}}, \quad H_{\alpha}:=\iota_{\alpha}^{*} G_{\alpha} .
$$

By the definition,

$$
\left.G_{\alpha}\right|_{\pi_{\alpha}^{-1}(t)}=g_{t^{m_{\alpha}}},\left.\quad H_{\alpha}\right|_{p_{\alpha}^{-1}(t)}=\iota_{\alpha}^{*} g_{t^{m_{\alpha}}}, \quad g_{t^{m_{\alpha}}}:=\left.G\right|_{X_{t^{m_{\alpha}}}} .
$$

Finally define a metric of $W_{\alpha}$ by

$$
g_{W_{\alpha}}:=\left.H_{\alpha}\right|_{W_{\alpha}}
$$

and metrics of $Z_{\alpha}$ and $Z$ by

$$
g_{Z_{\alpha}}:=\left.\iota^{*} g_{0}\right|_{Y_{\alpha}}=\left(\hat{g}_{\alpha}\right)^{*} g_{W_{\alpha}}=\left(\iota_{\alpha} \circ \hat{g}_{\alpha}\right)^{*} g_{0}, \quad g_{Z} \mid Z_{\alpha}:=g_{Z_{\alpha}} .
$$

The goal of this section is to prove the following theorem.

TheOREM 1.1. For every small $0<\epsilon \ll 1$, there exists $0<\gamma(\epsilon)<\epsilon$ and a family of into-diffeomorphisms for $|t|<\gamma(\epsilon)$ :

$$
f_{\epsilon, t}: Z-S_{\epsilon} \hookrightarrow X_{t}
$$

by which the following conditions are satisfied.

(1) On $Z-S_{\epsilon}$,

$$
\frac{1}{2} g_{Z} \leq f_{\epsilon, t}^{*} g_{t} \leq 2 g_{Z}
$$

(2) On every compact subset $K \Subset Z-S \epsilon$,

$$
\lim _{t \rightarrow 0} f_{\epsilon, t}^{*} g_{t}=g_{Z}
$$


in the $C^{\infty}$-topology on $K$.

(3) If $\operatorname{vol}(\cdot)$ stands for the volume relative to the induced metric from $G$ and $g_{Z}$, then

$$
\operatorname{vol}\left(X_{t}-f_{\epsilon, t}\left(Z-S_{\epsilon}\right)\right) \leq 3 \operatorname{vol}\left(S_{\epsilon}\right)
$$

For the proof, we need some lemmas and propositions.

LEMMA 1.1. For every small $0<\epsilon \ll 1$, there exist an open neighborhood $U_{\alpha}(\epsilon)$ of $W_{\alpha}-\Sigma_{\alpha, \epsilon}$ in $\mathfrak{X}_{\alpha}$, and vector fields $u_{\alpha}^{(\epsilon)}$, $v_{\alpha}^{(\epsilon)}$ on $U_{\alpha}(\epsilon)$ such that

$$
\left(p_{\alpha}\right)_{*} u_{\alpha}^{(\epsilon)}=\frac{\partial}{\partial x}, \quad\left(p_{\alpha}\right)_{*} v_{\alpha}^{(\epsilon)}=\frac{\partial}{\partial y}
$$

where $t_{\alpha}=x+\sqrt{-1} y$.

Proof. Clear by Proposition 1.1 and the compactness of $W_{\alpha}-\Sigma_{\alpha, \epsilon}$ in $\mathfrak{X}_{\alpha}$.

Lemma 1.2. For every small $0<\epsilon \ll 1$, there exist $0<\gamma_{1}(\epsilon)<\epsilon$ and an into-diffeomorphism

$$
\Phi_{\alpha}^{(\epsilon)}:\left(W_{\alpha}-\Sigma_{\alpha, \epsilon}\right) \times \Delta\left(\gamma_{1}(\epsilon)\right) \longrightarrow U_{\alpha}(\epsilon)
$$

such that

(1) For any $t \in \Delta\left(\gamma_{1}(\epsilon)\right)$,

$$
\Phi_{\alpha}^{(\epsilon)}\left(W_{\alpha}-\Sigma_{\alpha, \epsilon}, t\right) \subset p_{\alpha}^{-1}(t) .
$$

(2) $\Phi_{\alpha}^{(\epsilon)}(\cdot, 0)$ is the identity map on $W_{\alpha}-\Sigma_{\alpha, \epsilon}$.

Proof. Integrating the vector fields in Lemma 1.1, desired into-diffeomorphism is obtained.

Proposition 1.2. For every small $0<\epsilon \ll 1$, there exist $0<\gamma_{2}(\epsilon)<$ $\epsilon$ and a family of into-diffeomorphisms for $|t|<\gamma_{2}(\epsilon)$

$$
\phi_{\alpha, t}^{(\epsilon)}: W_{\alpha}-\Sigma_{\alpha, \epsilon} \rightarrow X_{t^{m_{\alpha}}}
$$


such that

(1) $\phi_{\alpha, 0}^{(\epsilon)}$ is the identity map of $W_{\alpha}-\Sigma_{\alpha, \epsilon}$.

(2) On $W_{\alpha}-\Sigma_{\alpha, \epsilon}$,

$$
\frac{1}{2} g_{W_{\alpha}} \leq\left(\phi_{\alpha, t}^{(\epsilon)}\right)^{*} g_{t^{m_{\alpha}}} \leq 2 g_{W_{\alpha}}
$$

(3) On $W_{\alpha}-\Sigma_{\alpha, \epsilon}$,

$$
\lim _{t \rightarrow 0}\left(\phi_{\alpha, t}^{(\epsilon)}\right)^{*} g_{t^{m_{\alpha}}}=g_{W_{\alpha}}
$$

in the $C^{\infty}$-topology on $W_{\alpha}-\Sigma_{\alpha, \epsilon}$.

Proof. For $|t|<\gamma_{1}(\epsilon)$, set

$$
\phi_{\alpha, t}^{(\epsilon)}(x):=\iota_{\alpha} \circ \Phi_{\alpha}^{(\epsilon)}(x, t)
$$

where $\gamma_{1}(\epsilon)$ and $\Phi_{\alpha}^{(\epsilon)}$ are the same ones as in Lemma 1.2. Then, $\phi_{\alpha, t}^{(\epsilon)}$ is a map from $W_{\alpha}-\Sigma_{\alpha, \epsilon}$ to $\iota_{\alpha} \circ\left(p_{\alpha}^{-1}(t)\right)=\pi_{\alpha}^{-1}(t)=X_{t^{m_{\alpha}}}$. By the definition, $\iota_{\alpha}: p_{\alpha}^{-1}(t) \rightarrow \pi_{\alpha}(t)$ is an isomorphism for $t \neq 0$. By Lemma 1.2, $\Phi_{\alpha}^{(\epsilon)}(\cdot, t)$ : $W_{\alpha}-\Sigma_{\alpha, \epsilon} \rightarrow \pi_{\alpha}^{-1}(t)$ is an into-diffeomorphism for $|t|<\gamma_{1}(\epsilon)$. Namely, $\phi_{\alpha, t}^{(\epsilon)}$ is an into-diffeomorphism from $W_{\alpha}-\Sigma_{\alpha, \epsilon}$ to $X_{t^{m_{\alpha}}}$.

By (1.31) and (1.32), we get

$$
\begin{aligned}
\left(\phi_{\alpha, t}^{(\epsilon)}\right)^{*} g_{t^{m_{\alpha}}} & =\left.\left(\left.\iota_{\alpha} \circ \Phi_{\alpha}^{(\epsilon)}\right|_{t}\right)^{*} G_{\alpha}\right|_{\pi_{\alpha}^{-1}(t)} \\
& =\left(\left.\Phi_{\alpha}^{(\epsilon)}\right|_{t}\right)^{*} H_{\alpha} .
\end{aligned}
$$

Since $\Phi_{\alpha}^{(\epsilon)}$ depends smoothly on $t$, it follows that

$$
\begin{aligned}
\lim _{t \rightarrow 0}\left(\left.\Phi_{\alpha}^{(\epsilon)}\right|_{t}\right)^{*} H_{\alpha} & =\left(\left.\Phi_{\alpha}^{(\epsilon)}\right|_{0}\right)^{*} H_{\alpha} \\
& =\left.H_{\alpha}\right|_{p_{\alpha}^{-1}(0)}=g_{W_{\alpha}} .
\end{aligned}
$$

(3) follows from (1.37). Since $W_{\alpha}-\Sigma_{\alpha, \epsilon}$ is compact, there exists $\gamma_{2}(\epsilon) \leq$ $\gamma_{1}(\epsilon)$ such that $(2)$ holds for $|t|<\gamma_{2}(\epsilon)$.

Proof of Theorem 1.1. For every $m_{\alpha}$, let $t^{\frac{1}{m_{\alpha}}}$ be a fixed branch of the inverse function of $t^{m_{\alpha}}$ on $\Delta(1)$. Let $\phi_{\alpha, t}^{(\epsilon)}$ be the same map as in Proposition 1.2. Since $Z-S_{\epsilon}=\cup_{\alpha}\left(Z_{\alpha}-S_{\alpha, \epsilon}\right)$, to define $f_{\epsilon, t}: Z-S_{\epsilon} \rightarrow X_{t}$, it is sufficient to define it on each $Z_{\alpha}-S_{\alpha, \epsilon}$. Define $f_{\epsilon, t}$ by

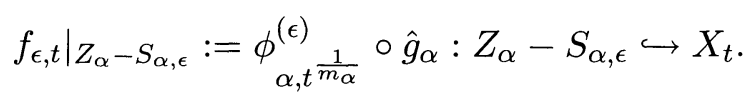


Since $\hat{g}: Z-S_{\epsilon} \rightarrow \bigcup_{\alpha}\left(W_{\alpha}-S_{\alpha, \epsilon}\right)$ is an isomorphism by Proposition 1.1, $f_{\epsilon, t}$ is an into-diffeomorphism for every small $t$ with $|t|<\gamma_{2}(\epsilon)$. Then we get (1) and (2) by Proposition 1.2 , because $\lim _{t \rightarrow 0} t^{\frac{1}{m_{\alpha}}}=0$ although $t^{\frac{1}{m_{\alpha}}} \notin C^{0}(\Delta(1))$.

By Proposition 1.2, for every small $0<\epsilon \ll 1$, there exists $\gamma_{3}(\epsilon)$ such that

$$
\left|\operatorname{vol}\left(f_{\epsilon, t}\left(Z-S_{\epsilon}\right)\right)-\operatorname{vol}\left(Z-S_{\epsilon}\right)\right|<\operatorname{vol}\left(S_{\epsilon}\right)
$$

for every $t$ with $|t|<\gamma_{3}(\epsilon)$. Since $\operatorname{vol}\left(Z-S_{\epsilon}\right)=\operatorname{vol}(Z)-\operatorname{vol}\left(S_{\epsilon}\right)=\operatorname{vol}\left(X_{0}\right)-$ $\operatorname{vol}\left(S_{\epsilon}\right)$, it follows from $(1.39)$

$$
\operatorname{vol}\left(f_{\epsilon, t}\left(Z-S_{\epsilon}\right)\right)>\operatorname{vol}\left(X_{0}\right)-2 \operatorname{vol}\left(S_{\epsilon}\right)
$$

for $t$ with $|t|<\gamma_{3}(\epsilon)$. Let $\omega_{G}$ be the Kähler form of $G$. Then,

$$
\operatorname{vol}\left(X_{t}\right):=\int_{X_{t}} \omega_{G}^{n}
$$

By [F, Proposition 2.3], as $\operatorname{vol}\left(X_{t}\right)$ is a continious function in $t$, for every small $0<\epsilon \ll 1$, there exists $\gamma_{4}(\epsilon)$ such that

$$
\left|\operatorname{vol}\left(X_{t}\right)-\operatorname{vol}\left(X_{0}\right)\right|<\operatorname{vol}\left(S_{\epsilon}\right)
$$

for every $t$ with $|t|<\gamma_{4}(\epsilon)$. By (1.40) and (1.42), for $t$ with $|t|<$ $\min \left\{\gamma_{3}(\epsilon), \gamma_{4}(\epsilon)\right\}$,

$$
\begin{aligned}
\operatorname{vol}\left(X_{t}-f_{\epsilon, t}\left(Z-S_{\epsilon}\right)\right) & =\operatorname{vol}\left(X_{t}\right)-\operatorname{vol}\left(f_{\epsilon, t}\left(Z-S_{\epsilon}\right)\right) \\
& <\operatorname{vol}\left(X_{t}\right)-\operatorname{vol}\left(X_{0}\right)+2 \operatorname{vol}\left(S_{\epsilon}\right) \\
& <3 \operatorname{vol}\left(S_{\epsilon}\right) .
\end{aligned}
$$

Set $\gamma(\epsilon):=\min \left\{\gamma_{2}(\epsilon), \gamma_{3}(\epsilon), \gamma_{4}(\epsilon)\right\}$. Then, Theorem 1.1 is proved for this $\gamma(\epsilon)$.

\section{$\S 2$. Sobolev inequality and upper bound of the heat kernel}

In this section, we recall some basic results on the Sobolev inequality and the heat kernel. For the reference, see [D].

Let $(M, g)$ be a compact Riemannian manifold of dimension $m$ with possibly smooth boundary. We denote by $\Delta$ the Laplacian and by $k(t, x, y)$ the heat kernel. When $M$ has boundary, consider the Dirichlet Laplacian and the Dirichlet heat kernel. As an application of the logarithmic Sobolev inequality, the following result is well known. 
Theorem 2.1. ([C-K-S] and [D, Theorem 2.4.2]) For $\mu>2$, the following two inequalities are equivalent:

(1) For $0<t \leq 1$ and $(x, y) \in M \times M$,

$$
k(t, x, y) \leq C_{1} t^{-\frac{\mu}{2}} .
$$

(2) For every $f \in C^{\infty}(M)$,

$$
\|f\|_{\frac{2 \mu}{\mu-2}} \leq C_{2}\left(\|d f\|_{2}+\|f\|_{2}\right) .
$$

Here $C_{1}$ and $C_{2}$ depend continuously on each other.

Let $\left\{0=\lambda_{0}<\lambda_{1} \leq \lambda_{2} \cdots\right\}$ be the eigenvalue of the Laplacian and $\phi_{i}$ be the normalized eigenfunction such that

$$
\Delta \phi_{i}=\lambda_{i} \phi_{i}, \quad\left(\phi_{i}, \phi_{j}\right)=\delta_{i j}
$$

By [C-L, Corollary 1], the following estimate holds.

Proposition 2.1. Under the two inequalities of Theorem 2.1,

$$
\left\|\phi_{i}\right\|_{\infty} \leq C_{3} \lambda_{i}^{\frac{\mu}{2}}
$$

where $C_{3}$ depends on $C_{1}$ and $C_{2}$.

In the sequel of this article (in §5), we shall use Theorem 2.1 and Proposition 2.1 for $\mu=m$ when $m>2$ and $\mu=4$ when $m=2$.

\section{$\S 3$. The space of functions on algebraic varieties}

Let $\left(X, g_{X}\right)$ be an irreducible projective algebraic variety of dimension $n$ in $\mathbb{P}^{N}(\mathbb{C})$ with the Bergmann metric, i.e. the restriction of the standard Fubini-Study metric of $\mathbb{P}^{N}(\mathbb{C})$. We denote by $\Sigma_{X}$ the singular set of $X$.

Let $C^{1 / 2}(X)$ (resp. $\left.C_{0}^{1 / 2}(X)\right)$ be the space of all Lipschitz functions (resp. with compact support on $X-\Sigma_{X}$ ) on $X$. Define $W^{1,2}(X)$ and $W_{0}^{1,2}(X)$ by

$$
\begin{gathered}
W^{1,2}(X):=\overline{\left\{f \in C^{1 / 2}(X) ; f \in L^{2}(X), d f \in L^{2}(X)\right\}} \\
W_{0}^{1,2}(X):=\overline{C_{0}^{1 / 2}(X)}
\end{gathered}
$$


where the completion is taken with respect to the norm $\|\cdot\|_{L_{1}^{2}}$ :

$$
\|f\|_{L_{1}^{2}}=\|f\|_{2}+\|d f\|_{2}
$$

In $[\mathrm{L}-\mathrm{T}]$, it is proved that $W^{1,2}(X)=W_{0}^{1,2}(X)$. Since the proof seems to be rough, we shall give a detailed proof in this section.

Let us fix notations. For any closed subset $S \subset \mathbb{P}^{N}(\mathbb{C})$, set

$$
\begin{aligned}
& r_{S}(x):=d(x, S)=\inf _{y \in S} d(x, y), \\
& S_{\epsilon}:=\left\{x \in \mathbb{P}^{N}(\mathbb{C}) ; r_{S}(x)<\epsilon\right\}
\end{aligned}
$$

where $d(\cdot, \cdot)$ is the distance of $\mathbb{P}^{N}(\mathbb{C})$.

THEOREM 3.1. Let $Y$ be a subvariety of pure dimension $d(<n)$ in $X$. Then, for the pair $(X, Y)$, there exist functions $\gamma(\cdot), \delta(\cdot)(\geq 0)$ $\in C^{0}([0,1 / 16])$ and $\eta_{\epsilon} \in C_{0}^{0}(X-Y) \cap W^{1,2}(X)$ for any $\epsilon \in(0,1 / 16]$ which satisfy the following conditions:

$$
0 \leq \eta_{\epsilon} \leq 1, \quad\left\|\eta_{\epsilon}-1\right\|_{2}+\left\|d \eta_{\epsilon}\right\|_{2} \leq \gamma(\epsilon), \quad \lim _{\epsilon \rightarrow 0} \gamma(\epsilon)=0
$$

$$
\begin{aligned}
& X-Y_{\delta(\epsilon)} \subset \operatorname{supp} \eta_{\epsilon} \subset X-Y_{\epsilon}, \quad \operatorname{supp}\left(1-\eta_{\epsilon}\right) \subset Y_{2 \delta(\epsilon)} \\
& \epsilon \leq \delta(\epsilon), \quad \lim _{\epsilon \rightarrow 0} \delta(\epsilon)=0 .
\end{aligned}
$$

THEOREM 3.2. There exist functions $\delta(\cdot)(\geq 0) \in C^{0}([0,1 / 16])$ and $\bar{\eta}_{\epsilon} \in C_{0}^{0}\left(X-\Sigma_{X}\right) \cap W^{1,2}(X)$ for any $\epsilon \in(0,1 / 16]$ which satisfy the following conditions:

(1)

$$
\operatorname{supp} \bar{\eta}_{\epsilon} \subset X-\Sigma_{\frac{\epsilon}{2}}, \quad 0 \leq \bar{\eta}_{\epsilon} \leq 1+\epsilon
$$

$$
\lim _{\epsilon \rightarrow 0}\left\|\bar{\eta}_{\epsilon}-1\right\|_{2}+\left\|d \bar{\eta}_{\epsilon}\right\|_{2}=0
$$

(3) If we set $S(\epsilon):=\left\{x \in X ; \bar{\eta}_{\epsilon}(x)=0\right\}$, then $\partial S(\epsilon)$ consists of finitely 
many smooth manifolds.

(4)

$$
\Sigma_{\frac{\epsilon}{2}} \subset S(\epsilon) \subset \Sigma_{\delta(\epsilon)}, \quad \lim _{\epsilon \rightarrow 0} \delta(\epsilon)=0 .
$$

In view of [L-T, Theorem 4.1], we get the following corollary.

COROLlARY 3.1. For any projective algebraic variety of pure dimension, $W^{1,2}(X)=W_{0}^{1,2}(X)$. Equivalently, $d_{\max }=d_{\min }$ on $L^{2}(X)$.

For the proof, we prepare several lemmas and propositions.

We denote by $Y_{\text {reg }}:=Y-\Sigma_{Y}$ the regular part of $Y$. For $y \in Y_{\text {reg }}$, set

$$
N_{y}:=\exp _{y}\left(T_{y} Y\right)^{\perp} \subset \mathbb{P}^{N}(\mathbb{C})
$$

where the exponential map is considered in $\mathbb{P}^{N}(\mathbb{C})$ relative to the FubiniStudy metric. Then $N_{y}$ is isometric to $\mathbb{P}^{N-d}(\mathbb{C})$ and intersect $Y$ transversally at $y$. Therefore there is a neighborhood $U$ of $y$ such that $Y \cap N_{y} \cap U=$ $\{y\}$. Let

$$
N_{y} \cap X=\cup_{\alpha}\left(N_{y} \cap X\right)_{\alpha}
$$

be the irreducible decomposition. Set

$$
\left(N_{y} \cap X\right)_{y}:=\bigcup_{\beta ; y \in\left(N_{y} \cap X\right)_{\beta}}\left(N_{y} \cap X\right)_{\beta}
$$

At first let us study the dimension of $\left(N_{y} \cap X\right)_{y}$. Since each $\left(N_{y} \cap X\right)_{\alpha}$ is a pure dimensional space, it follows that

$$
\begin{aligned}
& \operatorname{dim}\left(N_{y} \cap X\right)_{\alpha}=\operatorname{dim}_{y}\left(N_{y} \cap X\right)_{\alpha}, \\
& \operatorname{dim}\left(N_{y} \cap X\right)_{y}=\max _{\alpha} \operatorname{dim}_{y}\left(N_{y} \cap X\right)_{\alpha}
\end{aligned}
$$

where $\operatorname{dim}_{y} W$ stands for the analytic dimension of $W$ at $y$ for algebraic variety $W$. See [G-R, Chap. 5] for the definition of dimension.

Proposition 3.1. There exists a nonempty Zariski open subset $U_{Y}$ of $Y$ such that if $y \in U_{Y}$,

$$
\operatorname{dim}_{y} N_{y} \cap X=n-d
$$


Proof. See Appendix Proposition A.1.

Since $\operatorname{dim}\left(N_{y} \cap X\right)_{\alpha} \geq n-d$ by the intersection inequality ([G-R, p. 102]), we get the following proposition.

Proposition 3.1'. If $y \in U_{Y}$,

$$
\operatorname{dim}\left(N_{y} \cap X\right)_{y}=n-d
$$

Next let us study the volume of $\left(N_{y} \cap X\right)_{y}$. For an algebraic variety $W \subset \mathbb{P}^{N}(\mathbb{C})$, its volume with respect to the Fubini-Study metric is given by

$$
\operatorname{vol}(W)=\left.\int_{W}\left(\frac{\sqrt{-1}}{2} \omega\right)^{l}\right|_{W},
$$

where $\omega$ is the Kähler form of the ambient projective space and $l=\operatorname{dim} W$. Since $\frac{\sqrt{-1}}{2 \pi} \omega$ represents the same current as $H$, a hyperplane in the ambient projective space (cf. $[\mathrm{G}-\mathrm{H}]$ ), we have

$$
\operatorname{vol}(W)=(\pi)^{l} \operatorname{deg}(W), \quad \operatorname{deg}(W)=\sharp\left(W \cap H_{1} \cap \cdots \cap H_{l}\right),
$$

where $H_{1}, \cdots, H_{l}$ are generic hyperplanes.

Proposition 3.2. Let $H_{1}, \cdots, H_{r}$ be arbitrary $r(<n)$-th hyperplanes in $\mathbb{P}^{N}(\mathbb{C})$. Let

$$
X \cap H_{1} \cap \cdots \cap H_{r}=\sum_{i=0}^{r} X_{r}^{(n-i)} \quad\left(\operatorname{dim} X_{r}^{(n-i)}=n-i\right)
$$

be the decomposition into the pure dimensional components. Then,

$$
\operatorname{deg}(X) \geq \operatorname{deg}\left(X_{r}^{(n-r)}\right) .
$$

Proof. See Appendix Proposition A.2.

Since $N_{y}$ is a linear subspace of dimension $N-d$ in the ambient projective space, there are hyperplanes $H_{1}, \cdots, H_{d}$ such that

$$
N_{y}=H_{1} \cap \cdots \cap H_{d}
$$

By Proposition 3.2, we get the following proposition. 
Proposition 3.2'. If $y \in U_{Y}$,

$$
\operatorname{deg}\left(N_{y} \cap X\right)_{y} \leq \operatorname{deg}(X)
$$

Let us study the tubular neighborhood of $Y$ in $\mathbb{P}^{N}(\mathbb{C})$. If $Y$ is smooth, it is diffeomorphic to the normal bundle of $Y$. Define the normal projective bundle $N_{Y}$ by

$$
N_{Y}:=\left\{(y, w) \in Y_{\text {reg }} \times \mathbb{P}^{N}(\mathbb{C}) ; w \in N_{y}\right\} .
$$

We denote by $i: N_{Y} \hookrightarrow Y_{\text {reg }} \times \mathbb{P}^{N}(\mathbb{C})$ the inclusion. Define projections by

$$
\pi:=p_{1} \circ i: N_{Y} \longrightarrow Y_{\mathrm{reg}}, \quad p:=p_{2} \circ i: N_{Y} \longrightarrow \mathbb{P}^{N}(\mathbb{C})
$$

where $p_{i}$ stands for the projection to the $i$-th factor. It is clear that $\pi$ : $N_{Y} \rightarrow Y_{\text {reg }}$ is a fiber bundle with fiber $\mathbb{P}^{N-d}(\mathbb{C})$. Define a subbundle of the tangent bundle of $N_{Y}$ by

$$
T N:=\operatorname{Ker}\left(\pi_{*}: T N_{Y} \rightarrow T Y_{\text {reg }}\right) .
$$

Then for $(y, w) \in N_{Y}$, it follows that $T N_{w}=T_{w} N_{y}$. By this identification, define a Riemannian metric $g_{N}$ of $T N$ by

$$
g_{N}:=\bigcup_{y \in Y_{\mathrm{reg}}} g_{N_{y}}
$$

where $g_{N_{y}}:=\left.g_{\mathbb{P}^{N}}\right|_{N_{y}}$ is a Riemannian metric of $N_{y}$. Define a ball bundle $N_{Y}(\epsilon)$ by

$$
N_{Y}(\epsilon):=\left\{(y, w) \in N_{Y} ; d_{\mathbb{P}^{N}}(y, w)<\epsilon\right\} .
$$

LEMMA 3.1. Let $S$ be a subvariety of $Y$ such that $S \supset \operatorname{Sing}(Y)$ and $\operatorname{dim} S<\operatorname{dim} Y$. Then, for the pair $(Y, S)$, there exists an increasing function $a(\epsilon) \in C^{0}([0,1])$ which satisfies the following conditions:

$$
\lim _{\epsilon \rightarrow 0} a(\epsilon)=0, \quad a(\epsilon) \geq \sqrt{\epsilon}
$$

(2) $p: \pi^{-1}\left(Y-S_{a(\epsilon)}\right) \cap N_{Y}(\epsilon) \rightarrow \mathbb{P}^{N}(\mathbb{C})$ is an into-diffeomorphism such that

$$
\frac{1}{2} p^{*} g_{\mathbb{P}^{N}} \leq \pi^{*} g_{Y}+g_{N} \leq 2 p^{*} g_{\mathbb{P}^{N}}
$$

on $\pi^{-1}\left(Y-S_{a(\epsilon)}\right) \cap N_{Y}(\epsilon)$ where $g_{Y}:=\left.g_{\mathbb{P}^{N}}\right|_{Y}$. 
Proof. By the definition of $p$, it is the identity map restricted to $Y_{\text {reg }}$. Choose a point $y \in Y_{\text {reg. }}$. By the definition of $N_{y}$, we get the following decomposition of the tangent space of $\mathbb{P}^{N}(\mathbb{C})$ and $N_{Y}$ at $y$ :

$$
T_{y} \mathbb{P}^{N}(\mathbb{C})=T_{y} N_{Y}=T_{y} Y \oplus T_{y} N_{y}, \quad T_{y} Y \perp T_{y} N_{y} .
$$

Choose tangent vectors $u \in T_{y} Y$ and $v \in T_{y} N_{y}$ arbitrarily. Since $\left.p\right|_{Y}=\mathrm{id}$ as above, we know

$$
\left(p_{*}\right)_{y} u=u \text {. }
$$

Choose a curve $\gamma(t):=\left(y, \exp _{y}(t v)\right)$ in $N_{Y}$. By the definition,

$$
\left(p_{*}\right)_{y} v=\left.\frac{d}{d t}\right|_{t=0} p(\gamma(t))=\left.\frac{d}{d t}\right|_{t=0} \exp _{y}(t v)=v
$$

This shows $\left(p_{*}\right)_{y}=1$. Since $y$ is an arbitrary point of $Y_{\text {reg }}$, we get $p_{*}=1$ along $Y_{\text {reg. Set }}$

$$
\begin{aligned}
b(\epsilon):=\sup \{b & \in(0,1] ; \\
p & \left.: \pi^{-1}\left(Y-S_{\epsilon}\right) \cap N_{Y}(b) \rightarrow \mathbb{P}^{N}(\mathbb{C}) \text { is an embedding }\right\} .
\end{aligned}
$$

By (3.18) and (3.19), we get on $Y_{\text {reg }}$

$$
p^{*} g_{\mathbb{P}^{N}}=\pi^{*} g_{Y}+g_{N} .
$$

In the same way as the definition of $b(\epsilon)$, set

$$
\begin{aligned}
c(\epsilon):= & \sup \{c \in(0,1\} ; \\
& \left.\frac{1}{2} p^{*} g_{\mathbb{P}^{N}} \leq \pi^{*} g_{Y}+g_{N} \leq 2 p^{*} g_{\mathbb{P}^{N}} \text { on } \pi^{-1}\left(Y-S_{\epsilon}\right) \cap N_{Y}(c)\right\} .
\end{aligned}
$$

By the definition, $b(\epsilon)$ and $c(\epsilon)$ are increasing functions and satisfy $b(\epsilon)$, $c(\epsilon)<1$. Since $Y-S_{\epsilon}$ is compact, $b(\epsilon), c(\epsilon)>0$.

Define $B(\epsilon)$ by

$$
B(\epsilon):=\int_{0}^{\epsilon} \min \{b(t), c(t)\} t d t \leq \frac{\epsilon^{2}}{2} \min \{b(\epsilon), c(\epsilon)\} .
$$

Then $p: \pi^{-1}\left(Y-S_{\epsilon}\right) \cap N_{Y}(B(\epsilon)) \rightarrow \mathbb{P}^{N}(\mathbb{C})$ is an into-diffeomorphism and the inequality of Lemma 3.1 (2) holds on $\pi^{-1}\left(Y-S_{\epsilon}\right) \cap N_{Y}(B(\epsilon)$ ). Furthermore, $B(\epsilon)$ is a continuous increasing function satisfying

$$
\lim _{\epsilon \rightarrow 0} B(\epsilon)=0 \text {. }
$$


Finally, define $a(\epsilon)$ by the inverse function of $B(\epsilon)$ :

$$
a(\epsilon):=B^{-1}(\epsilon)(\geq \sqrt{\epsilon}) .
$$

By (3.23) and (3.24), $a(\epsilon)$ is a continuous increasing function satisfying Lemma 3.1 (1).

By (3.22) and (3.23), the inequality of Lemma 3.1 (2) is satisfied by $a(\epsilon)$.

LEMMA 3.2. Let $\mathbb{B}(r):=\left\{z=\left(z_{1}, \cdots, z_{N}\right) \in \mathbb{C}^{N} ;\|z\|^{2}:=\sum_{i=1}^{N}\left|z_{i}\right|^{2}\right.$ $<r\}$ be the ball of radius $r$ in $\mathbb{C}^{N}$. Then the following inequality holds:

$$
-\partial \bar{\partial} \log \left(-\log \|z\|^{2}\right) \geq \frac{\partial \bar{\partial}\|z\|^{2}}{\|z\|^{2}\left(-\log \|z\|^{2}\right)^{2}}
$$

for $z \in \mathbb{B}(1 / 2)$ where $\partial \bar{\partial} \phi$ is the complex Hessian:

$$
\partial \bar{\partial} \phi:=\sum_{i, j=1}^{N} \frac{\partial^{2} \phi}{\partial z_{i} \partial \bar{z}_{j}} d z_{i} d \bar{z}_{j} .
$$

Proof. By computations, we get

$$
-\partial \bar{\partial} \log \left(-\log \|z\|^{2}\right)=\sum_{i, j=1}^{N} G_{i \bar{j}}(z) d z_{i} d \bar{z}_{j}
$$

$$
G_{i \bar{j}}(z)=\frac{\delta_{i j}}{\|z\|^{2}\left(-\log \|z\|^{2}\right)}-\frac{\bar{z}_{i} z_{j}}{\|z\|^{4}\left(-\log \|z\|^{2}\right)}+\frac{\bar{z}_{i} z_{j}}{\|z\|^{4}\left(-\log \|z\|^{2}\right)^{2}},
$$

which implies

$$
G(z):=\left(G_{i \bar{j}}\right) \geq \frac{1}{\|z\|^{2}\left(-\log \|z\|^{2}\right)^{2}} I .
$$

LEMMA 3.3. Let $V \subset \mathbb{P}^{N}(\mathbb{C})$ be a projective algebraic variety of pure dimension $d$. Let $U$ be the standard affine open subset of $\mathbb{P}^{N}(\mathbb{C})$; i.e., $U=$ $\mathbb{C}^{N}$. Let $\left(z_{1}, \cdots, z_{N}\right)$ be the coordinate of $U$. Then the following inequality holds for any $\epsilon \in(0,1)$ :

$$
\int_{\mathbb{B}(\epsilon) \cap V} d v_{V} \leq C(N) \epsilon^{2 d} \operatorname{deg}(V)
$$

where $C(N)$ is a constant which depends only on $N$, and $d v_{V}$ is the volume form of $V$ with respect to the Bergmann metric of $V$. 
Proof. By the definition,

$$
d v_{V}=\left.\left(\omega^{d}\right)\right|_{V}
$$

where $\omega$ is the Kähler form of $\mathbb{P}^{N}(\mathbb{C})$. We denote by $\Omega$ the Kähler form of $\mathbb{C}^{N}$ with respect to the Euclidean metric. On $\mathbb{B}(1)$,

$$
\frac{1}{2} \Omega \leq \omega \leq \Omega
$$

Let $T_{\epsilon}$ be a linear transform of $\mathbb{C}^{N}$ defined by

$$
T_{\epsilon}(z):=\epsilon z
$$

We denote by $\tilde{T}_{\epsilon}$ the extension of $T_{\epsilon}$ to $\mathbb{P}^{N}(\mathbb{C})$. Since

$$
\int_{\mathbb{B}(\epsilon) \cap V} \Omega^{d}=\epsilon^{2 d} \int_{\mathbb{B}(1) \cap \tilde{T}_{\epsilon^{-1}}(V)} \Omega^{d}
$$

it follows from (3.9), (3.10) and (3.30)

$$
\int_{\mathbb{B}(\epsilon) \cap V} d v_{V} \leq(4 \pi)^{d} \epsilon^{2 d} \operatorname{deg}\left(\tilde{T}_{\epsilon^{-1}}(V)\right) .
$$

Since $\tilde{T}_{\epsilon^{-1}} \in \operatorname{Aut}\left(\mathbb{P}^{N}(\mathbb{C})\right)$, we find

$$
\operatorname{deg}(V)=\operatorname{deg}\left(\tilde{T}_{\epsilon^{-1}}(V)\right)
$$

which combined with (3.33) yields the assertion.

LEMMA 3.4. Let $C$ be an algebraic curve in $\mathbb{P}^{N}(\mathbb{C})$. Let $U$ be the affine open set and $z=\left(z_{1}, \cdots, z_{N}\right)$ be the coordinate of $U$ as in the previous lemma. Set

$$
\rho(z):=d_{\mathbb{P}^{N}}(0, z)=\int_{0}^{\|z\|} \frac{d r}{1+r^{2}} .
$$

Then, for any $\epsilon \in(0,1 / 16)$, the following inequality holds:

$$
\int_{C \cap\{\epsilon \leq \rho \leq \sqrt{\epsilon}\}} \frac{d v_{C}}{\rho^{2}(-\log \epsilon)^{2}} \leq C(N) \frac{\operatorname{deg}(C)}{-\log \epsilon} .
$$


Proof. In this proof, we consider $\partial \bar{\partial} \phi$ as a 2-form for any function $\phi$. By the definition,

$$
\frac{1}{2}\|z\| \leq \rho(z) \leq\|z\| \quad \text { for } \rho \leq \sqrt{\epsilon} .
$$

Therefore by Lemma 3.2,

$$
\begin{aligned}
\int_{C \cap\{\epsilon \leq \rho \leq \sqrt{\epsilon}\}} & \frac{d v_{C}}{\rho^{2}(-\log \epsilon)^{2}} \\
& \leq 2 \int_{C \cap\{\epsilon \leq r \leq 2 \sqrt{\epsilon}\}} \frac{\sqrt{-1}}{2} \frac{\partial \bar{\partial}\|z\|^{2}}{\|z\|^{2}\left(-\log \|z\|^{2}\right)^{2}} \\
& \leq 2 \int_{C \cap\{\epsilon \leq r \leq 2 \sqrt{\epsilon}\}}-\frac{\sqrt{-1}}{2} \partial \bar{\partial} \log \left(-\log \|z\|^{2}\right)
\end{aligned}
$$

where $r(z):=\|z\|$. Set

$$
d^{c}:=-\sqrt{-1}(\partial-\bar{\partial}), \quad d d^{c}=2 \sqrt{-1} \partial \bar{\partial} .
$$

By computation,

$$
\begin{aligned}
I(\epsilon) & :=\int_{C \cap\{\epsilon \leq r \leq 2 \sqrt{\epsilon}\}}-\frac{\sqrt{-1}}{2} \partial \bar{\partial} \log \left(-\log \|z\|^{2}\right) \\
& =\frac{1}{4} \int_{C \cap\{\epsilon \leq r \leq 2 \sqrt{\epsilon}\}}-d d^{c} \log \left(-\log \|z\|^{2}\right) \\
& =\frac{1}{4} \int_{C \cap\{r=2 \sqrt{\epsilon}\}}-d^{c} \log \left(-\log \|z\|^{2}\right)-\frac{1}{4} \int_{C \cap\{r=\epsilon\}}-d^{c} \log \left(-\log \|z\|^{2}\right) \\
(3.38) & =\frac{1}{4} \int_{C \cap\{r=2 \sqrt{\epsilon}\}} \frac{d^{c}\|z\|^{2}}{\|z\|^{2}\left(-\log \|z\|^{2}\right)}-\frac{1}{4} \int_{C \cap\{r=\epsilon\}} \frac{d^{c}\|z\|^{2}}{\|z\|^{2}\left(-\log \|z\|^{2}\right)} \\
& =\frac{1}{4} \int_{C \cap\{r=2 \sqrt{\epsilon}\}} \frac{d^{c}\|z\|^{2}}{(2 \sqrt{\epsilon})^{2}\left(-\log (2 \sqrt{\epsilon})^{2}\right)}-\frac{1}{4} \int_{C \cap\{r=\epsilon\}} \frac{d^{c}\|z\|^{2}}{\epsilon^{2}\left(-\log \epsilon^{2}\right)} \\
& =(2 \sqrt{\epsilon})^{-2}\left(-\log (2 \sqrt{\epsilon})^{2}\right)^{-1} \int_{C \cap \mathbb{B}(2 \sqrt{\epsilon})} \frac{\sqrt{-1}}{2} \partial \bar{\partial}\|z\|^{2} \\
& -\epsilon^{-2}\left(-\log \epsilon^{2}\right)^{-1} \int_{C \cap \mathbb{B}(\epsilon)} \frac{\sqrt{-1}}{2} \partial \bar{\partial}\|z\|^{2} .
\end{aligned}
$$

Applying Lemma 3.3 to the integrands of the last equality of (3.38), we get

$$
\begin{aligned}
& \int_{C \cap\{\epsilon \leq r \leq 2 \sqrt{\epsilon}\}}-\frac{\sqrt{-1}}{2} \partial \bar{\partial} \log \left(-\log \|z\|^{2}\right) \\
& \leq C(N) \operatorname{deg}(C)\left(\frac{1}{-\log 4 \epsilon}+\frac{1}{-\log \epsilon^{2}}\right) .
\end{aligned}
$$


When $\epsilon \in(0,1 / 16)$, it is easy to verify the inequality:

$$
\frac{1}{-\log 4 \epsilon}+\frac{1}{-\log \epsilon^{2}} \leq\left(\log \frac{1}{\epsilon}\right)^{-1}
$$

By (3.36), (3.38), (3.39) and (3.40), we get the desired inequality.

Proof of Theorem 3.1. We prove the theorem by induction. When $\operatorname{dim} Y \leq \operatorname{dim} X-2$, define a function $\rho_{Y, \epsilon}$ by

$$
\rho_{Y, \epsilon}(x):= \begin{cases}0 & \left(r_{Y}(x) \leq \epsilon\right) \\ \epsilon^{-1}\left(r_{Y}(x)-\epsilon\right) & \left(\epsilon \leq r_{Y}(x) \leq 2 \epsilon\right) \\ 1 & \left(r_{Y}(x) \geq 2 \epsilon\right),\end{cases}
$$

where $r_{Y}$ is the same function as (3.3). When $\operatorname{dim} Y=\operatorname{dim} X-1$, define $\rho_{Y, \epsilon}$ by

$$
\rho_{Y, \epsilon}(x):= \begin{cases}0 & \left(r_{Y}(x) \leq \epsilon\right) \\ \frac{2}{\log \epsilon^{-1}} \int_{\epsilon}^{r_{Y}(x)} \frac{d \rho}{\rho} & \left(\epsilon \leq r_{Y}(x) \leq \sqrt{\epsilon}\right) \\ 1 & \left(r_{Y}(x) \geq \sqrt{\epsilon}\right) .\end{cases}
$$

It is clear by the definition that $0 \leq \rho_{Y, \epsilon} \leq 1$.

First let us prove the theorem when $\operatorname{dim} X>1$ and $\operatorname{dim} Y=0$. Set $\gamma(\epsilon)=C \epsilon, \delta(\epsilon)=2 \epsilon$ and $\eta_{\epsilon}(x):=\rho_{Y, \epsilon}(x)$. By the definition (3.41), the condition (2) of the theorem is satisfied. Since $Y$ is a discrete set of $X$, we may assume $Y=\{p\}$. By computation,

$$
\begin{aligned}
\left\|1-\eta_{\epsilon}\right\|_{2}^{2}+\left\|d \eta_{\epsilon}\right\|_{2}^{2} & \leq \operatorname{vol}\left(X \cap Y_{\epsilon}\right)+\epsilon^{-2} \int_{\epsilon \leq r_{Y} \leq 2 \epsilon}\left|d r_{Y}\right|^{2} d v_{X} \\
& \leq C(N) \operatorname{deg}(X) \epsilon^{2 n}+\sup _{X}\left|d r_{Y}\right|^{2} \epsilon^{-2} \operatorname{vol}\left(X \cap Y_{2 \epsilon}\right) \\
& \leq C(N) \operatorname{deg}(X) \epsilon^{2(n-1)}\left(1+\sup _{X}\left|d r_{Y}\right|^{2}\right)
\end{aligned}
$$

where Lemma 3.3 is used. By the definition of $r_{Y}$, we find

$$
\left|r_{Y}(x)-r_{Y}(y)\right| \leq d_{\mathbb{P}^{N}}(x, y) \leq d_{X}(x, y)
$$

for any $x, y \in X$ which implies

$$
\sup _{X}\left|d r_{Y}\right| \leq \sqrt{2 n}
$$


Commbining (3.43) and (3.44), we get

$$
\left\|1-\eta_{\epsilon}\right\|_{2}^{2}+\left\|d \eta_{\epsilon}\right\|_{2}^{2} \leq C(N) \operatorname{deg}(X) \epsilon^{2(n-1)},
$$

which proves the theorem when $\operatorname{dim} Y=0$.

Next assume the theorem for any subvariety of pure dimension $k-1$ and prove it for an arbitrary subvariety $Y$ of pure dimension $k$. Let $U_{Y}$ be the Zariski open subset of $Y$ considered in Proposition 3.1. Set

$$
Z=Z_{Y}:=\left(Y-U_{Y}\right) \cup \operatorname{Sing}(Y)
$$

Then $Z$ is a subvariety of $Y$ whose dimension is strictly smaller than $k$.

Let $Z=\cup_{i} Z_{i}$ be the irreducible decomposition of $Z$. By the induction hypothesis, there exists a function $\eta_{i, \epsilon}$ which satisfies the conditions of Theorem 3.1 for $\left(X, Z_{i}\right)$. Set

$$
\xi_{\epsilon}(x):=\prod_{i} \eta_{i, \epsilon}(x)
$$

Let $\gamma_{i}(\epsilon)$ and $\delta_{i}(\epsilon)$ be the functions of Thorem 3.1 for $\left(X, Z_{i}\right)$. Set

$$
\gamma_{Z}(\epsilon):=\sharp\{i\} \max _{i} \gamma_{i}(\epsilon), \quad \delta_{Z}(\epsilon):=\max _{i} \delta_{i}(\epsilon) .
$$

Then it is easily verified that $\gamma_{Z}(\epsilon), \delta_{Z}(\epsilon)$ and $\xi_{\epsilon}$ satisfy the conditions of Theorem 3.1 for the pair $(X, Z)$. Therefore $\left\{\xi_{\epsilon}\right\}$ is a family of functions which satisfies the conditions of Theorem 3.1 for $(X, Z)$.

Since $Z \supset \operatorname{Sing}(Y)$, we can apply Lemma 3.1 to the pair $(Y, Z)$. Let $a(\epsilon)$ be the function considered in the lemma. Define $\eta_{\epsilon}$ by

$$
\eta_{\epsilon}(x):=\rho_{Y, \epsilon}(x) \xi_{2 a(2 \epsilon)}(x) .
$$

In the sequel, we shall verify the conditions of Theorem 3.1 for $\left\{\eta_{\epsilon}\right\}$.

By the definition, it is clear

$$
0 \leq \eta_{\epsilon} \leq 1
$$

Next we verify (2). Since

$$
\begin{aligned}
& \operatorname{supp} \eta_{\epsilon}=\operatorname{supp} \rho_{\epsilon} \cap \operatorname{supp} \xi_{2 a(2 \epsilon)}, \quad \operatorname{supp} \rho_{\epsilon}=X-Y_{\epsilon}, \\
& \bigcup_{i}\left(X-Z_{i, \delta_{i}(\epsilon)}\right) \subset \operatorname{supp} \xi_{\epsilon} \subset \bigcup_{i}\left(X-Z_{i, \epsilon}\right),
\end{aligned}
$$


we have

$$
\left(X-Y_{\epsilon}\right) \cap \cup_{i}\left(X-Z_{i, \delta_{i}(2 a(2 \epsilon))}\right) \subset \operatorname{supp} \eta_{\epsilon} \subset\left(X-Y_{\epsilon}\right) \cap \cup_{i}\left(X-Z_{i, \epsilon}\right) .
$$

As $Z$ is a subset of $Y$ and $\delta_{Z}(2 a(2 \epsilon)) \geq \max _{i}\left\{\delta_{i}(2 a(2 \epsilon)), \epsilon\right\}$, it is clear

$$
X-Y_{\delta_{Z}(2 a(2 \epsilon))} \subset \operatorname{supp} \eta_{\epsilon} \subset X-Y_{\epsilon} \text {. }
$$

Since

$$
\begin{aligned}
\operatorname{supp}\left(1-\eta_{\epsilon}\right) \subset \operatorname{supp}(1- & \left.\rho_{\epsilon}\right) \cup \operatorname{supp}\left(1-\xi_{2 a(2 \epsilon)}\right) \\
& \subset \operatorname{supp}\left(1-\rho_{\epsilon}\right) \cup \bigcup_{i} \operatorname{supp}\left(1-\eta_{i, 2 a(2 \epsilon)}\right)
\end{aligned}
$$

when $k=\operatorname{dim} Y<n-1$, we get

$$
\operatorname{supp}\left(1-\eta_{\epsilon}\right) \subset Y_{2 \epsilon} \cup \bigcup_{i} Z_{i, 2 a(2 \epsilon)} \subset Y_{2 \delta_{Z}(2 a(2 \epsilon))}
$$

and when $k=\operatorname{dim} Y=n-1$,

$$
\operatorname{supp}\left(1-\eta_{\epsilon}\right) \subset Y_{\sqrt{\epsilon}} \cup Z_{\delta_{Z}(2 a(2 \epsilon))}
$$

Set

$$
\tilde{\delta}(\epsilon):=\max \left\{\sqrt{\epsilon}, \delta_{Z}(2 a(2 \epsilon))\right\}(\geq \epsilon)
$$

By (3.54), (3.57) and (3.58), Theorem 3.1 (2) is satisfied by $\eta_{\epsilon}$ and $\tilde{\delta}(\epsilon)$.

Next verify the latter part of (1).

Case $1(k=\operatorname{dim} Y<n-1)$

From (3.50) and the induction hypothesis, it follows that

$$
\begin{aligned}
\left\|1-\eta_{\epsilon}\right\|_{2} & \leq\left\|1-\rho_{\epsilon}\right\|_{2}+\left\|1-\xi_{2 a(2 \epsilon)}\right\|_{2} \\
& \leq \sqrt{\operatorname{vol}\left(Y_{2 \epsilon}\right)}+\gamma_{Z}(2 a(2 \epsilon))
\end{aligned}
$$

and

$$
\begin{aligned}
\left\|d \eta_{\epsilon}\right\|_{2} & =\left\|\xi_{2 a(2 \epsilon)} d \rho_{\epsilon}+\rho_{\epsilon} d \xi_{2 a(2 \epsilon)}\right\|_{2} \\
& \leq\left\|\xi_{2 a(2 \epsilon)} d \rho_{\epsilon}\right\|_{2}+\left\|d \xi_{2 a(2 \epsilon)}\right\|_{2} \\
& \leq\left\|\xi_{2 a(2 \epsilon)} d \rho_{\epsilon}\right\|_{2}+\gamma_{Z}(2 a(2 \epsilon)) .
\end{aligned}
$$


It follows from $(3.41)$,

$$
\begin{aligned}
\left\|\xi_{2 a(2 \epsilon)} d \rho_{\epsilon}\right\|_{2}^{2} & =\epsilon^{-2} \int_{X \cap\left(Y_{2 \epsilon}-Y_{\epsilon}\right) \cap \operatorname{supp} \xi_{2 a(2 \epsilon)}}\left|d r_{Y}\right|^{2} d v_{X} \\
& \leq 2 n \epsilon^{-2} \operatorname{vol}\left(\left(Y_{2 \epsilon}-Y_{\epsilon}\right) \cap\left(X-Z_{2 a(2 \epsilon)}\right)\right)
\end{aligned}
$$

When $x \in\left(Y_{2 \epsilon}\right) \cap\left(X-Z_{2 a(2 \epsilon)}\right)$, let $y$ be a point of $Y$ such that $r_{Y}(x)=$ $d_{\mathbb{P}^{N}}(x, y)$. By the trigonometrical inequality and (3.25), we get

$$
\begin{aligned}
d_{\mathbb{P} N}(y, Z) & \geq d_{\mathbb{P}^{N}}(x, Z)-d_{\mathbb{P}^{N}}(x, y) \\
& \geq 2 a(2 \epsilon)-2 \epsilon \\
& \geq a(2 \epsilon)
\end{aligned}
$$

which implies $y \in Y-Z_{a(2 \epsilon)}$ and $x \in N_{y}(2 \epsilon)$. Therefore we have

$$
\left(Y_{2 \epsilon}-Y_{\epsilon}\right) \cap\left(X-Z_{2 a(2 \epsilon)}\right) \subset p\left(\pi^{-1}\left(Y-Z_{a(2 \epsilon)}\right) \cap N_{Y}(2 \epsilon)\right)
$$

where $N_{Y}(\epsilon)$ is the same as in (3.16) and $p$ and $\pi$ are the same as in (3.13).

From (3.61), (3.63), Lemma 3.1 and Fubini's theorem, it follows that

$$
\begin{aligned}
\left\|\xi_{2 a(2 \epsilon)} d \rho_{\epsilon}\right\|_{2}^{2} & \leq 2 n \epsilon^{-2} \operatorname{vol}\left(X \cap p\left(\pi^{-1}\left(Y-Z_{a(2 \epsilon)}\right) \cap N_{Y}(2 \epsilon)\right)\right) \\
& \leq 4^{n} 2 n \epsilon^{-2} \int_{Y-Z_{a(2 \epsilon)}} d v_{Y} \int_{X \cap N_{y}(2 \epsilon)} d v_{X \cap N_{y}(2 \epsilon)}
\end{aligned}
$$

Since $y \in U_{Y},\left(X \cap N_{y}\right)_{y}$ is a pure dimensional space and thus Lemma 3.3 is applicable. Set $W_{y}:=\left(X \cap N_{y}\right)_{y}$ and consider $y$ as the origin of $U$ in Lemma 3.3. Applying Lemma 3.3 and Proposition 3.2, we get

$$
\begin{aligned}
\int_{X \cap N_{y}(2 \epsilon)} d v_{X \cap N_{y}(2 \epsilon)} & =\int_{W_{y} \cap\{\rho \leq 4 \epsilon\}} d v_{\mathbb{P}^{n-k}} \\
& \leq C(n-k) \operatorname{deg}\left(W_{y}\right)(8 \epsilon)^{2(n-k)} \\
& \leq C(N) \operatorname{deg}(X) \epsilon^{2(n-k)}
\end{aligned}
$$

which combined with (3.60), (3.64) yields

$$
\left\|d \eta_{\epsilon}\right\|_{2}^{2} \leq C(N) \operatorname{deg}(X) \epsilon^{2(n-k-1)} \operatorname{vol}(Y)+\gamma_{Z}(2 a(2 \epsilon))
$$

By (3.60) and (3.66), Theorem 3.1 (1) is proved for $\eta_{\epsilon}$. 
Set

$$
\tilde{\gamma}(\epsilon):=C(N) \operatorname{deg}(X) \epsilon^{2(n-k-1)}+\gamma_{Z}(2 a(2 \epsilon)) .
$$

From (3.49), (3.58) and the induction hypothesis, it follows that

$$
\lim _{\epsilon \rightarrow 0} \tilde{\gamma}(\epsilon)=0,
$$

which combined with (3.66) yields, the latter part of (1).

Case $2(k=\operatorname{dim} Y=n-1)$

By (3.59) and (3.60), it is sufficient to show

$$
\lim _{\epsilon \rightarrow 0}\left\|\xi_{2 a(2 \epsilon)} d \rho_{\epsilon}\right\|_{2}=0 .
$$

It follows from (3.42),

$$
\begin{aligned}
\left\|\xi_{2 a(2 \epsilon)} d \rho_{\epsilon}\right\|_{2}^{2} & =4\left(\log \epsilon^{-1}\right)^{-2} \int_{X \cap\left(Y_{\sqrt{\epsilon}}-Y_{\epsilon}\right) \cap \operatorname{supp} \xi_{2 a(2 \epsilon)}} r_{Y}^{-2}\left|d r_{Y}\right|^{2} d v_{X} \\
& \leq 8 n\left(\log \epsilon^{-1}\right)^{-2} \int_{X \cap\left(Y_{\sqrt{\epsilon}}-Y_{\epsilon}\right) \cap \operatorname{supp} \xi_{2 a(2 \epsilon)}} r_{Y}^{-2} d v_{X} .
\end{aligned}
$$

When $x \in\left(Y_{\sqrt{\epsilon}}\right) \cap\left(X-Z_{2 a(2 \epsilon)}\right)$, let $y$ be a point of $Y$ such that $r_{Y}(x)=$ $d_{\mathbb{P}^{N}}(x, y)$. By the trigonometrical inequality and (3.25), we get

$$
\begin{aligned}
d_{\mathbb{P}^{N}}(y, Z) & \geq d_{\mathbb{P}^{N}}(x, Z)-d_{\mathbb{P}^{N}}(x, y) \\
& \geq 2 a(2 \epsilon)-\sqrt{\epsilon} \\
& \geq a(\epsilon),
\end{aligned}
$$

which implies

$$
X \cap\left(Y_{\sqrt{\epsilon}}-Y_{\epsilon}\right) \cap \operatorname{supp} \xi_{3 a(\epsilon)} \subset p\left(\pi^{-1}\left(Y-Z_{a(\epsilon)}\right) \cap\left\{N_{Y}(\sqrt{\epsilon})-N_{Y}(\epsilon)\right\}\right) .
$$

From (3.70), (3.72), Lemma 3.1 and Fubini's theorem, it follows that

$$
\begin{aligned}
& \left\|\xi_{3 a(\epsilon)} d \rho_{\epsilon}\right\|_{2}^{2} \\
& \leq 8 n\left(\log \epsilon^{-1}\right)^{-2} \int_{X \cap p\left(\pi^{-1}\left(Y-Z_{a(\epsilon)}\right) \cap\left\{N_{Y}(\sqrt{\epsilon})-N_{Y}(\epsilon)\right\}\right)} r_{Y}^{-2} d v_{X} \\
& \leq 4^{n} 8 n\left(\log \epsilon^{-1}\right)^{-2} \int_{p^{-1}(X) \cap \pi^{-1}\left(Y-Z_{a(\epsilon)}\right) \cap\left(N_{Y}(\sqrt{\epsilon})-N_{Y}(\epsilon)\right)} r_{Y}^{-2} \pi^{*} d v_{Y} d v_{X \cap N_{Y}} \\
& =4^{n} 8 n\left(\log \epsilon^{-1}\right)^{-2} \int_{Y-Z_{a(\epsilon)}} d v_{Y}(y) \int_{\left(X \cap N_{y}\right)_{y} \cap\left(N_{y}(\sqrt{\epsilon})-N_{y}(\epsilon)\right)} r_{Y}^{-2} d v_{X \cap N_{y}} .
\end{aligned}
$$


Set $C_{y}:=\left(X \cap N_{y}\right)_{y}$. By $(3.73)$, Lemma 3.4 and Proposition 3.2,

$$
\begin{aligned}
\left\|\xi_{2 a(2 \epsilon)} d \rho_{\epsilon}\right\|_{2}^{2} & \leq 4^{n} 8 n \int_{Y-Z_{a(\epsilon)}} d v_{Y}(y) \int_{C_{y} \cap\left\{\epsilon \leq \rho_{y} \leq \sqrt{\epsilon}\right\}}\left(\log \epsilon^{-1}\right)^{-2} \rho_{y}^{-2} d v_{C_{y}} \\
& \leq \int_{Y-Z_{a(\epsilon)}} C(N) \operatorname{deg}\left(C_{y}\right)\left(\log \epsilon^{-1}\right)^{-1} d v_{Y}(y) \\
& \leq C(N) \operatorname{deg}(X) \operatorname{vol}(Y)\left(\log \epsilon^{-1}\right)^{-1}
\end{aligned}
$$

which yields (3.69). Set

$$
\tilde{\gamma}(\epsilon):=C(N) \operatorname{deg}(X) \operatorname{vol}(Y)\left(\log \epsilon^{-1}\right)^{-1}+\gamma_{Z}(2 a(2 \epsilon))
$$

Then

$$
\left\|1-\eta_{\epsilon}\right\|_{2}^{2}+\left\|d \eta_{\epsilon}\right\|_{2}^{2} \leq \tilde{\gamma}(\epsilon), \quad \lim _{\epsilon \rightarrow 0} \tilde{\gamma}(\epsilon)=0
$$

which proves the latter part of (1). As in the proof of Case 2, we can prove the theorem when $n=1$ and leave it to the reader.

Proof of Theorem 3.2. Let $\Sigma_{X}=\cup_{\alpha} \Sigma_{\alpha}$ be the irreducible decomposition. By Theorem 3.1, we may assume that for the pair $\left(X, \Sigma_{\alpha}\right)$, there exists a family of cut-off functions $\left\{\eta_{\alpha, \epsilon}\right\}_{\epsilon \in(0,1 / 16)}$ which satisfies the conditions of Theorem 3.1. Fix such a family and set

$$
\eta_{\epsilon}:=\prod_{\alpha} \eta_{\alpha, \epsilon}
$$

Then we find $\operatorname{supp} \eta_{\epsilon} \subset X-\Sigma_{\epsilon}$ by Theorem 3.1 (3). Since the boundaries of the zero set of each $\eta_{\epsilon}$ are not smooth by the construction, we must regularize the cut-off functions. For simplicity, set $\Sigma=\Sigma_{X}$.

Let $i(x)>0$ be the injectivity radius at $x \in X-\Sigma$. Put

$$
i(\epsilon):=\frac{1}{4} \min \left\{\inf _{x \in X-\Sigma_{\epsilon}} i(x), \epsilon\right\}
$$

Since $X-\Sigma_{\epsilon}$ is compact, $i(\epsilon)>0$ for $\epsilon>0$. Let $\left\{U_{i}\right\}_{i=1}^{L}$ be an open covering of $X-\Sigma_{\epsilon}$ chosen in such a way that $\bigcup_{i} U_{i} \subset X-\Sigma_{\epsilon / 2}$ and for each $U_{i}$, there is a metric ball $B_{i(\epsilon)}\left(x_{i}\right)$ of radius $i(\epsilon)$ centered at $x_{i}$ such that $U_{i}=B_{i(\epsilon)}\left(x_{i}\right)$ and $x_{i} \in X-\Sigma_{\epsilon}$. By the Gauss lemma, identify $U_{i}$ 
with the ball of radius $i(\epsilon)$ in the Eucilidean space of dimension $2 n$ via the exponential map

$$
\phi_{i}: \mathbb{B}(r) \rightarrow U_{i}=B_{r}\left(x_{i}\right)
$$

for all $r \leq 2 i(\epsilon)$. Since $i(\epsilon) \leq i\left(x_{i}\right)$, there is a constant $C_{i}>1$ such that, on $\mathbb{B}(i(\epsilon))$,

$$
C_{i}^{-1} g_{E} \leq \phi_{i}^{*} g_{X} \leq C_{i} g_{E}
$$

where $g_{E}$ is the standard Euclidean metric. Let $\left\{h_{i}\right\}$ be a partition of unity subject to the covering $\left\{U_{i}\right\}$ of $X-\Sigma_{\epsilon}$. Since $\eta_{\epsilon}=\sum_{i} h_{i} \eta_{\epsilon}$, first consider the regularization of $h_{i} \eta_{\epsilon}$. Set $\eta_{i, \epsilon}:=\phi_{i}^{*}\left(h_{i} \eta_{\epsilon}\right)$. Since $\eta_{\epsilon} \in C_{0}^{0}\left(X-\Sigma_{\epsilon}\right) \cap W^{1,2}(X)$, it follows that $\eta_{i, \epsilon} \in C_{0}^{0}\left(\mathbb{B}(i(\epsilon)) \cap W^{1,2}(\mathbb{B}(i(\epsilon))\right.$.

Following [G-H, Chap. 3, $\S 1]$, let $\chi \in C_{0}^{\infty}\left(\mathbb{R}^{2 n}\right)$ be a nonnegative function supported in a neighborfood of the origin with

$$
\int_{\mathbb{R}^{2 n}} \chi(x) d v=1
$$

Put

$$
\chi_{\delta}(x):=\delta^{-2 n} \chi\left(\frac{x}{\delta}\right) .
$$

If $\operatorname{supp} \chi=K$, then $\operatorname{supp} \chi_{\delta}=\delta K$ and

$$
\int_{\mathbb{R}^{2 n}} \chi_{\delta}(x) d v=1
$$

For any function $f \in L_{\text {loc }}^{1}\left(\mathbb{R}^{2 n}\right)$, set

$$
f_{\delta}(x):=\int_{\mathbb{R}^{2 n}} \chi_{\delta}(x-y) f(y) d y=\int_{\mathbb{R}^{2 n}} f(x-y) \chi_{\delta}(y) d y
$$

By [G-H, pp. 374] and [Na, Lemma 14.1], if $f \in C_{0}^{0}(\mathbb{B}(r)) \cap W^{1,2}\left(\mathbb{R}^{2 n}\right)$, then $\left\{f_{\delta}\right\}$ satisfies the following conditions:

(1) $f_{\delta}$ is a smooth function and $\operatorname{supp} f_{\delta} \subset \mathbb{B}(r+\delta)$.

$$
\lim _{\delta \rightarrow 0} \sup _{\mathbb{B}(2 r)}\left|f_{\delta}-f\right|=0
$$


(3)

$$
\lim _{\delta \rightarrow 0}\left\|f_{\delta}-f\right\|_{2}+\left\|d f_{\delta}-d f\right\|_{2}=0
$$

Set

$$
\eta_{\epsilon, \delta}:=\sum_{i}\left(\phi^{-1}\right)^{*}\left(\eta_{i, \epsilon}\right)_{\delta}
$$

From (3.79), Theorem 3.1 (1) and (1) above, if $\delta<i(\epsilon)$, it follows that $\eta_{\epsilon, \delta} \in C_{0}^{\infty}\left(X-\Sigma_{\epsilon / 2}\right)$. Since

$$
\sup _{X-\Sigma_{\epsilon / 2}}\left|\eta_{\epsilon, \delta}-\eta_{\epsilon}\right| \leq \sum_{i} \sup _{U_{i}}\left|\left(\eta_{i, \epsilon}\right)_{\delta}-\eta_{i, \epsilon}\right|
$$

$$
\left\|\eta_{\epsilon, \delta}-\eta_{\epsilon}\right\|_{2}+\left\|d \eta_{\epsilon, \delta}-d \eta_{\epsilon}\right\|_{2} \leq \sum_{i}\left\|\left(\eta_{i, \epsilon}\right)_{\delta}-\eta_{i, \epsilon}\right\|_{2}+\left\|d\left(\eta_{i, \epsilon}\right)_{\delta}-d \eta_{i, \epsilon}\right\|_{2}
$$

using (3.79), (3.85) and (3.86), there exists $j(\epsilon)$ such that $0<j(\epsilon)<i(\epsilon)$ and that for any $\delta \leq j(\epsilon)$,

$$
\begin{gathered}
\sup _{X-\Sigma_{\epsilon / 2}}\left|\eta_{\epsilon, \delta}-\eta_{\epsilon}\right|<\epsilon, \\
\left\|\eta_{\epsilon, \delta}-\eta_{\epsilon}\right\|_{2}+\left\|d \eta_{\epsilon, \delta}-d \eta_{\epsilon}\right\|_{2}<\epsilon .
\end{gathered}
$$

By the definition, $\eta_{\epsilon, j(\epsilon)}$ is a smooth function which takes zero and is not identically equal to zero. By Sard's theorem, there exists a regular value $A(\epsilon)$ of $\eta_{\epsilon, j(\epsilon)}$ such that $0<A(\epsilon)<\epsilon$. Finally, set

$$
\bar{\eta}_{\epsilon}(x):= \begin{cases}\eta_{\epsilon, j(\epsilon)}(x)-A(\epsilon) & \left(\eta_{\epsilon, j(\epsilon)}(x) \geq A(\epsilon)\right) \\ 0 & \left(\eta_{\epsilon, j(\epsilon)}(x) \leq A(\epsilon)\right) .\end{cases}
$$

Let us verify that $\bar{\eta}_{\epsilon}$ satisfies the conditions of Theorem 3.2.

It is clear that $\bar{\eta}_{\epsilon} \geq 0$, and that

$$
\operatorname{supp} \bar{\eta}_{\epsilon} \subset \operatorname{supp} \eta_{\epsilon, j(\epsilon)} \subset X-\Sigma_{\frac{\epsilon}{2}}
$$

as $j(\epsilon)<i(\epsilon)$ and $\operatorname{supp} \eta_{\epsilon, \delta} \subset X-\Sigma_{\epsilon / 2}$ for $\delta<i(\epsilon)$. From (3.90) and Theorem 3.1 (1), it follows that

$$
\bar{\eta}_{\epsilon} \leq \eta_{\epsilon, j(\epsilon)} \leq 1+\epsilon
$$


which proves Theorem 3.2 (1). By (3.91) and (3.92),

$$
\begin{aligned}
\left\|\bar{\eta}_{\epsilon}-1\right\|_{2}+\left\|d \bar{\eta}_{\epsilon}\right\|_{2} & \leq\left\|\eta_{\epsilon, j(\epsilon)}-1\right\|_{2}+\left\|d \eta_{\epsilon, j(\epsilon)}\right\|_{2}+\epsilon \sqrt{\operatorname{vol}(X)} \\
& \leq\left\|\eta_{\epsilon}-1\right\|_{2}+\left\|d \eta_{\epsilon}\right\|_{2}+\epsilon(1+\sqrt{\operatorname{vol}(X)})
\end{aligned}
$$

By (3.77) and Theorem 3.1 (1), we get

$$
\left\|\eta_{\epsilon}-1\right\|_{2}+\left\|d \eta_{\epsilon}\right\|_{2} \leq \sum_{\alpha}\left\|\eta_{\alpha, \epsilon}-1\right\|_{2}+\left\|d \eta_{\alpha, \epsilon}\right\|_{2} \leq \sum_{\alpha} \gamma_{\alpha}(\epsilon)
$$

where $\gamma_{\alpha}(\epsilon)$ satisfies Theorem 3.1 (1) for the pair $\left(X, \Sigma_{\alpha}\right)$, which combined with (3.95) yields Theorem $3.2(2)$. By (3.92), it is clear that

$$
S(\epsilon)=\left\{x \in X ; \eta_{\epsilon, j(\epsilon)}(x) \leq A(\epsilon)\right\}, \quad \partial S(\epsilon)=\eta_{\epsilon, j(\epsilon)}^{-1}(A(\epsilon)) .
$$

As $A(\epsilon)$ is a regular value of $\eta_{\epsilon, j(\epsilon)}, \partial S(\epsilon)$ is a smooth $2 n$-1-dimensional manifold and consists of finitely many connected components by the compactness of $X$. This proves (3).

When $x \in \Sigma_{\frac{\epsilon}{2}}$, then $\eta_{\epsilon, j(\epsilon)}(x)=0$ and therefore $x \in S(\epsilon)$ by (3.92), which implies $\Sigma_{\frac{\epsilon}{2}} \subset S(\epsilon)$. When $x \in S(\epsilon)$, it follows that $\eta_{\epsilon, j(\epsilon)}(x) \leq$ $A(\epsilon) \leq \epsilon$ which combined with (3.90) yields $\eta_{\epsilon}(x)<2 \epsilon<1$. This implies $x \in \operatorname{supp}\left(1-\eta_{\epsilon}\right)$. By $(3.77)$, one of $\alpha$ satisfies $\eta_{\alpha, \epsilon}(x)<1$. Therefore we have

$$
S(\epsilon) \subset \bigcup_{\alpha} \operatorname{supp}\left(1-\eta_{\alpha, \epsilon}\right) \subset \bigcup_{\alpha} \Sigma_{\alpha, 2 \delta_{\alpha}(\epsilon)} \subset \Sigma_{\delta(\epsilon)}
$$

where $\delta(\epsilon):=2 \max _{\alpha}\left\{\delta_{\alpha}(\epsilon)\right\}$ and $\delta_{\alpha}(\epsilon)$ satisfies Theorem 3.1 (2) for the pair $\left(X, \Sigma_{\alpha}\right)$.

Since $\lim _{\epsilon \rightarrow 0} \delta(\epsilon)=0$, we get Theorem 3.2 (4) by (3.97).

For our later purpose (cf. $\S 6)$, we need the following.

LEMMA 3.5. Let $(X, g)$ be an irreducible algebraic variety in $\mathbb{P}^{N}(\mathbb{C})$ with the Bergmann metric. Let $\pi: X^{\prime} \rightarrow X-\Sigma_{X}$ be a m-sheeted covering of $X-\Sigma_{X}$. Then

$$
W_{0}^{1,2}\left(X^{\prime}, \pi^{*} g\right)=W^{1,2}\left(X^{\prime}, \pi^{*} g\right)
$$

and Theorem 3.2 holds for $\left(X^{\prime}, \pi^{*} g\right)$.

Proof. By [L-T], it is sufficient to show Theorem 3.2 for $\left(X^{\prime}, \pi^{*} g\right)$. Considering the pull back of the family of cut-off functions of Theorem 3.2 for $(X, g)$, it satisfies the conditions of Theorem 3.2 , since $\pi$ is a finite covering. 


\section{§4. A comparison theorem for the heat kernel of projective varieties}

In this section, we recall the results of [L-T] concerning the upper bound of the heat kernel of projective algebraic varieties with the Bergmann metric.

Let $M$ be a projective algebraic variety of pure dimension $n$ in the projective space $\mathbb{P}^{N}(\mathbb{C})$. Let $g$ be the Bergmann metric of $M$; i.e., the restriction of the Fubini-Study metric of $\mathbb{P}^{N}(\mathbb{C})$. Let $S$ be the singular set of $M$. Consider the Dirichlet Laplacian of $M-S$; i.e., the Friedrichs extension of the Laplacian on $C_{0}^{\infty}(M-S)$ which is defined by:

$$
\Delta:=\delta_{\max } d_{\min }
$$

Since $d_{\max }=d_{\min }$ on the space of functions (cf. [L-T, Theorem 4.1] and Corollary 3.1), we can ignore the Dirichlet boundary condition. As the semigroup generated by the Dirichlet Laplacian has a smooth kernel function denoted by $K_{M}(t, x, y)$. In particular, we denote by $K_{\mathbb{P}^{n}}(t, x, y)$ the heat kernel of complex projective space of dimension $n$ with the Fubini-Study metric.

Let $r(x, y)$ be the distance of two points $x$ and $y$ in $\mathbb{P}^{n}(\mathbb{C})$ relative to the Fubini-Study metric. By the symmetry on $\mathbb{P}^{n}(\mathbb{C})$,

$$
K_{\mathbb{P}^{n}}(t, x, y)=K_{\mathbb{P}^{n}}(t, r(x, y))
$$

where $K_{\mathbb{P}^{n}}(t, s)$ is a function of $t$ and $s$. Now we can state the result of Li-Tian.

THEOREM 4.1. ([L-T]) Let $(M, g)$ be a projective algebraic variety of pure dimension $n$ with the Bergmann metric. Then the following comparison theorem of the heat kernels holds for all $(t, x, y) \in[0, \infty) \times(M-S) \times$ $(M-S)$ :

$$
K_{M}(t, x, y) \leq K_{\mathbb{P}^{n}}\left(t, r_{\mathbb{P}^{N}}(x, y)\right)
$$

where $r_{\mathbb{P}^{N}}(x, y)$ is the distance from $x$ to $y$ in $\mathbb{P}^{N}(\mathbb{C})$.

Combining Theorem 4.1 and Theorem 2.1, we have the following corollary. 
Corollary 4.1. Let $(M, g)$ be the same as in Theorem 4.1. Then there is a constant $C(n)$ which depends only on $n$ such that for every $f \in$ $C_{0}^{\infty}(M-S)$,

$$
\begin{gathered}
\|f\|_{\frac{2 n}{n-2}} \leq C(n)\left(\|d f\|_{2}+\|f\|_{2}\right) \quad(n>1), \\
\|f\|_{4} \leq C(1)\left(\|d f\|_{2}+\|f\|_{2}\right) \quad(n=1) .
\end{gathered}
$$

COROLlary 4.2. Let $\pi: \mathfrak{X} \rightarrow \Delta(1)$ be a one parameter degenerating family of projective algebraic manifolds of dimension $n$ in a fixed projective space $\mathbb{P}^{N}(\mathbb{C})$ as in the introduction. Let $G$ be an arbitrary Riemannian metric of $\mathfrak{X}$. Set $X_{t}:=\pi^{-1}(t)$ and $g_{t}:=\left.G\right|_{X_{t}}$ for $t \in \Delta$. Then there exists a constant $C>0$ independent of $t \in \Delta(1 / 2)-\{0\}$ such that for every $f \in C^{\infty}\left(X_{t}\right)$, the following inequality holds:

$$
\begin{aligned}
\|f\|_{\frac{2 n}{n-2}, t} \leq C\left(\|d f\|_{2, t}+\|f\|_{2, t}\right) & (n>1) \\
\|f\|_{4, t} \leq C\left(\|d f\|_{2, t}+\|f\|_{2, t}\right) & (n=1)
\end{aligned}
$$

where $\|\cdot\|_{p, t}$ is the $L^{p}$-norm with respect to $g_{t}$.

Proof. Since $\left.\mathfrak{X}\right|_{\Delta(1 / 2)} \in \mathbb{P}^{N}(\mathbb{C}) \times \Delta(1)$, there is a constant $C_{0}$ such that

$$
C_{0}^{-1}\left(g_{\mathbb{P}^{N}(\mathbb{C})}+|d t|^{2}\right) \leq g \leq C_{0}\left(g_{\mathbb{P}^{N}(\mathbb{C})}+|d t|^{2}\right)
$$

on $\pi^{-1}(\Delta(1 / 2))$ where $g_{\mathbb{P}^{N}(\mathbb{C})}$ is the Fubini-Study metric of $\mathbb{P}^{N}(\mathbb{C})$. which implies

$$
C_{0}^{-1} g_{\mathbb{P}^{N}(\mathbb{C})}\left|X_{t} \leq g_{t} \leq C_{0} g_{\mathbb{P}^{N}(\mathbb{C})}\right| X_{t}
$$

By Corollary 4.1 and (4.4), we obtain the desired estimates.

\section{$\S 5$. Continuity of the spectrum in the parameter}

Let $(M, g)$ be a Riemannian manifold of dimension $n$ with finite volume, and let $\left\{\left(M_{i}, g_{i}\right)\right\}_{i \geq 1}$ be a sequence of compact Riemannian manifolds of dimension $n$. In this section, we shall show that the spectrum of the Laplacian of $\left(M_{i}, g_{i}\right)$, converges to that of the Dirichlet Laplacian of $(M, g)$ as $i \rightarrow \infty$ under certain conditions, which is an abstract version of our Main Theorem. Introduce the following condition for $(M, g)$. 
CONDITION. (C1) (1) There exists a sequence of cut-off functions $\left\{\rho_{i}\right\}_{i=0}^{\infty} \subset C_{0}^{0}(M) \cap W^{1,2}(M)$ which satisfies the following conditions:

$$
\lim _{i \rightarrow \infty}\left\|\rho_{i}-1\right\|_{2}+\left\|d \rho_{i}\right\|_{2}=0, \quad 0 \leq \rho_{i} \leq 1
$$

(2) $S_{i}:=\left\{x \in M ; \rho_{i}(x)=0\right\}$ has finitely many smooth boundaries.

$$
\lim _{i \rightarrow \infty} \operatorname{vol}\left(S_{i}\right)=0
$$

Set $\gamma(i):=\left\|\rho_{i}-1\right\|_{2}+\left\|d \rho_{i}\right\|_{2}$. Then $\gamma(i) \rightarrow 0$ as $i \rightarrow \infty$.

Remark 5.1. From the argument of [L-T, Theorem 4.1], under (C1), it follows that $W^{1,2}(M)=W_{0}^{1,2}(M)$ with respect to $g$, or equivalently $d_{\max }=d_{\min }$ for $(M, g)$.

Introduce the following condition for the family $\left\{\left(M_{i}, g_{i}\right)\right\}$ :

Condition. (C2) There exists a sequence of open subsets $\left\{S_{i}\right\}$ of $M$ and a sequence of into-diffeomorphisms $\left\{f_{i}\right\}_{i \geq 1} f_{i}: M-S_{i} \hookrightarrow M_{i}$ which satisfies the following conditions:

(1) For every $i \geq 1$,

$$
\frac{1}{2} g \leq f_{i}^{*} g_{i} \leq 2 g
$$

on $M-S_{i}$.

(2) If we trivially extend $f_{i}^{*} g_{i}$ to $M$ by setting $f_{i}^{*} g_{i}:=0$ on $S_{i}$, then

$$
\lim _{i \rightarrow \infty} f_{i}^{*} g_{i}=g
$$

almost everywhere on $M$.

(3) Setting $K_{i}:=M_{i}-f_{i}\left(M-S_{i}\right)$,

$$
\lim _{i \rightarrow \infty} \operatorname{vol}\left(K_{i}\right)=0 \text {. }
$$

If $\left\{S_{i}\right\}$ is the same one as in $(\mathrm{C} 1)$, then $(\mathrm{C} 2)$ is said to be subject to $(\mathrm{C} 1)$.

Let $\left\{\lambda_{1}(i) \leq \lambda_{2}(i) \leq \cdots\right\}$ be the spectrum of $\Delta_{i}$, the Laplacian of $\left(M_{i}, g_{i}\right)$, and $\left\{\lambda_{1} \leq \lambda_{2} \leq \cdots\right\}$ the spectrum of $\Delta:=\delta_{\max } d_{\min }$, the Dirichlet Laplacian of $(M, g)$, counted with multiplicities. Our goal in this section is to prove the following theorem. 
THEOREM 5.1. Let $\left\{\left(M_{i}, g_{i}\right)\right\}$ be a sequence of compact Riemannian manifolds and $(M, g)$ a Riemannian manifold with finite volume satisfying (C1). If (C2) subject to $(\mathrm{C} 1)$ is satisfied for $\left\{\left(M_{i}, g_{i}\right)\right\}$ and $(M, g)$, and if the Sobolev inequality is uniform; i.e., there exists a constant $C>0$ independent of $i$ such that

$$
\|\phi\|_{\frac{2 n}{n-2}, i} \leq C\left(\|d \phi\|_{2, i}+\|\phi\|_{2, i}\right)
$$

for every $\phi \in C^{\infty}\left(M_{i}\right)$ where $\|\cdot\|_{p, i}$ is the $L^{p}$-norm of $\left(M_{i}, g_{i}\right)$, then

$$
\lim _{i \rightarrow \infty} \lambda_{k}(i)=\lambda_{k}
$$

As mentioned in the introduction, we prove the theorem only when $n>2$. To show that the spectrum of the Dirichlet Laplacian of $M$ consists of discrete eigenvalues, we need the Rellich lemma for $M$.

LEMMA 5.1. Under the assumption of Theorem 5.1, the Sovolev inequality holds on $M$. Namely there exists a constant $C>0$ such that for any $\psi \in C_{0}^{\infty}(M)$, the following inequality holds:

$$
\|\psi\|_{\frac{2 n}{n-2}} \leq C\left(\|d \psi\|_{2}+\|\psi\|_{2}\right)
$$

Proof. Let $\psi \in C_{0}^{\infty}(M)$. Since $\left(f_{i}^{-1}\right)^{*}\left(\rho_{i} \psi\right)$ is a smooth function on $M_{i}$, we get by the Sobolev inequality on $M_{i}$ and $(\mathrm{C} 2)$,

$$
\left\|\rho_{i} \psi\right\|_{\frac{2 n}{n-2}} \leq 4^{n} C\left(\|d \psi\|_{2}+\|\psi\|_{2}+\|\psi\|_{\infty}\left\|d \rho_{i}\right\|_{2}\right)
$$

Since $\left\|d \rho_{i}\right\|_{2} \leq \gamma(i)$ by (C1), we obtain the desired inequality, taking the limit as $i \rightarrow \infty$.

Proposition 5.1. (Rellich lemma) Inclusion $W^{1,2}(M) \hookrightarrow L^{2}(M)$ is compact.

Proof. Let $\left\{f_{n}\right\}$ be a bounded sequence in $W^{1,2}(M)$; i.e.,

$$
\left\|f_{n}\right\|_{2}+\left\|d f_{n}\right\|_{2} \leq C_{0}<\infty
$$

for all $n$. Since $M-S_{i}$ has a smooth compact boundary, Rellich lemma holds for any $M-S_{i}$ (cf. [G-T, Theorem 7.26]). By the diagonalization 
argument, we can choose a subsequence $\left\{f_{n m}\right\}$ which converges in every $L^{2}\left(M-S_{i}\right)$. By the Hölder inequality, Lemma 5.1 and Remark 5.1, we get

$$
\begin{aligned}
\left\|f_{n m}\right\|_{L^{2}\left(S_{2}\right)} & \leq \operatorname{vol}\left(S_{i}\right)^{\frac{1}{n}}\left\|f_{n m}\right\|_{L^{\frac{2 n}{n-2}}\left(S_{i}\right)} \\
& \leq C \operatorname{vol}\left(S_{i}\right)^{\frac{1}{n}}\left(\left\|f_{n m}\right\|_{2}+\left\|d f_{n m}\right\|_{2}\right) \\
& \leq C C_{0} \operatorname{vol}\left(S_{i}\right)^{\frac{1}{n}} .
\end{aligned}
$$

Let $\epsilon>0$ be an arbitrary given number. By (C1), there exists $i(\epsilon)$ such that

$$
C C_{0} \operatorname{vol}\left(S_{i}\right)^{\frac{1}{n}}<\frac{\epsilon}{2} \quad \text { for } \quad i \geq i(\epsilon) .
$$

Therefore for $i \geq i(\epsilon)$, we get

$$
\left\|f_{n m}-f_{n m^{\prime}}\right\|_{2} \leq\left\|f_{n m}-f_{n m^{\prime}}\right\|_{L^{2}\left(M-S_{2}\right)}+\frac{\epsilon}{2} .
$$

Since $\left\{f_{n m}\right\}$ converges on $M-S_{i}$, there exists $m(\epsilon)$ such that if $m, m^{\prime} \geq$ $m(\epsilon)$,

$$
\left\|f_{n m}-f_{n m^{\prime}}\right\|_{L^{2}\left(M-S_{i}\right)}<\frac{\epsilon}{2}
$$

which combined with (5.3) yields $\left\|f_{n m}-f_{n m^{\prime}}\right\|_{2}<\epsilon$ for $m, m^{\prime} \geq m(\epsilon)$.

COROLlary 5.1. The spectrum of the Dirichlet Laplacian of $(M, g)$ consists of discrete eigenvalues.

Let $\left\{\phi_{k}(i)\right\}$ be a complete orthonormal system of $L^{2}\left(M_{i}\right)$ which consists of eigenfunctions of $\Delta_{i}$; i.e.,

$$
\Delta_{i} \phi_{k}(i)=\lambda_{k}(i) \phi_{k}(i), \quad\left(\phi_{k}(i), \phi_{l}(i)\right)_{i}=\delta_{k l}
$$

where $(\cdot, \cdot)_{i}$ stands for the inner product of $L^{2}\left(M_{i}\right)$. Set

$$
\psi_{k}(i):=\rho_{i} f_{i}^{*} \phi_{k}(i) \in C_{0}^{\infty}\left(M-S_{i}\right)
$$

From the definition, Proposition 2.1 and (C), it follows that for all $i$ and $k$

$$
\left\|\psi_{k}(i)\right\|_{2} \leq C^{\prime} \lambda_{k}(i)^{n}
$$

where $C^{\prime}$ is a constant depending only on $C$, the constant in Theorem 5.1. 
Proposition 5.2. For every $N \geq 0$, there exists a subsequence $\{i(\nu)\}$ such that the following formulae hold for $0 \leq k \leq N$ :

$$
\begin{gathered}
\lim _{\nu \rightarrow \infty} \lambda_{k}(i(\nu))=\lambda_{k} . \\
s-\lim _{\nu \rightarrow \infty} \psi_{k}(i(\nu))=\phi_{k} \quad \text { in } L^{2}(M) \\
w-\lim _{\nu \rightarrow \infty} \psi_{k}(i(\nu))=\phi_{k} \quad \text { in } W^{1,2}(M)
\end{gathered}
$$

where $\phi_{k}$ is the eigenfunction of $\Delta$ such that $\left(\phi_{k}, \phi_{l}\right)=\delta_{k l}$ and $\Delta \phi_{k}=\lambda_{k} \phi_{k}$.

For the proof, we need the following.

Lemma 5.2. Suppose that Proposition 5.2 is true for $N$. Then,

$$
\lim \sup _{i \rightarrow \infty} \lambda_{N+1}(i) \leq \lambda_{N+1}
$$

Proof. By Proposition 5.2 for $N$, we may assume

$$
\begin{array}{cc}
s-\lim _{i \rightarrow \infty} \psi_{k}(i)=\phi_{k} & \text { in } L^{2}(M) \\
w-\lim _{i \rightarrow \infty} \psi_{k}(i)=\phi_{k} & \text { in } W^{1,2}(M)
\end{array}
$$

for $0 \leq i \leq N$. Let $\phi_{N+1}$ be the eigenfunction of $\Delta_{0}$ such that

$$
\left(\phi_{i}, \phi_{N+1}\right)=\delta_{i, N+1} \quad \text { and } \quad \Delta_{0} \phi_{N+1}=\lambda_{N+1} \phi_{N+1}
$$

for $i \leq N+1$. Since $W^{1,2}(M)=W_{0}^{1,2}(M)$, there is a sequence $\left\{\phi_{N+1, i}\right\} \subset$ $C_{0}^{\infty}(M)$ such that

$$
\left\|\phi_{N+1, i}-\phi_{N+1}\right\|_{L_{1}^{2}} \leq \frac{1}{i}, \quad \operatorname{supp} \phi_{N+1, \nu} \subset M-S_{i} .
$$

Set

$$
\begin{gathered}
\chi_{N+1}(i):=\left(f_{i}^{-1}\right)^{*} \phi_{N+1, i} \in C_{0}^{\infty}\left(M_{i}-K_{i}\right), \\
\xi_{N+1}(i):=\chi_{N+1}(i)-\sum_{k \leq N}\left(\chi_{N+1}(i), \phi_{k}(i)\right)_{i} \phi_{k}(i),
\end{gathered}
$$




$$
P\left(\phi_{N+1, i}\right):=\xi_{N+1}(i) /\left\|\xi_{N+i}(i)\right\|_{2, i} \in C^{\infty}\left(M_{i}\right) .
$$

It follows from the definition

$$
\begin{gathered}
\left\|P\left(\phi_{N+1, i}\right)\right\|_{2, i}=1, \quad P\left(\phi_{N+1, i}\right) \perp\left\{\phi_{0}(i), \cdots, \phi_{N}(i)\right\}, \\
\left\|d P\left(\phi_{N+1, i}\right)\right\|_{2, i}^{2} \geq \inf _{f \perp\left\{\phi_{0}(i), \cdots, \phi_{N}(i)\right\}}\|d f\|_{2}^{2} /\|f\|_{2}^{2}=\lambda_{N+1}(i),
\end{gathered}
$$

(5.17) $\left\|d P\left(\phi_{N+1, i}\right)\right\|_{2, i}^{2}=\frac{\left\|d \chi_{N+1}(i)-\sum_{k \leq N}\left(\chi_{N+1}(i), \phi_{k}(i)\right)_{i} d \phi_{k}(i)\right\|_{2, i}^{2}}{\left\|\chi_{N+1}(i)-\sum_{k \leq N}\left(\chi_{N+1}(i), \phi_{k}(i)\right)_{i} \phi_{k}(i)\right\|_{2, i}^{2}}$.

By computation,

$$
\begin{aligned}
\left(\chi_{N+1}(i), \phi_{k}(i)\right)_{i} & =\left(\left(f_{i}^{-1}\right)^{*} \phi_{N+1, i}, \phi_{k}(i)\right)_{i} \\
& =\int_{M_{\imath}-K_{\imath}}\left(f_{i}^{-1}\right)^{*} \phi_{N+1, i} \phi_{k}(i) d v_{i} \\
& =\int_{M-S_{i}} \phi_{N+1, i} f_{i}^{*} \phi_{k}(i) f_{i}^{*} d v_{i} .
\end{aligned}
$$

Set

$$
G_{i}:= \begin{cases}f_{i}^{*} d v_{i} / d v & \left(M-S_{i}\right) \\ 0 & \left(S_{i}\right)\end{cases}
$$

By the definition and (C2), it follows that

$$
\left\|G_{i}\right\|_{\infty} \leq 2^{n}
$$

From (5.18) and (5.19), it follows that

$$
\begin{aligned}
\left(\chi_{N+1}(i), \phi_{k}(i)\right)_{i}=\left(\phi_{N+1, i}, f_{i}^{*} \phi_{k}(i) G_{i}\right) \\
=\left(\phi_{N+1, i}, \psi_{k}(i)\right)+\left(\phi_{N+1, i},\left(1-\rho_{i}\right) f_{i}^{*} \phi_{k}(i)\right) \\
+\left(\phi_{N+1, i}, f_{i}^{*} \phi_{k}(i)\left(G_{i}-1\right)\right) \\
=\left(\phi_{N+1}, \psi_{k}(i)\right)+\left(\phi_{N+1, i}-\phi_{N+1}, \psi_{k}(i)\right) \\
+\left(\phi_{N+1, i},\left(1-\rho_{i}\right) f_{i}^{*} \phi_{k}(i)\right) \\
+\left(\phi_{N+1, i}, f_{i}^{*} \phi_{k}(i)\left(G_{i}-1\right)\right) .
\end{aligned}
$$


By the hypothesis,

$$
\lim _{i \rightarrow \infty}\left(\phi_{N+1}, \psi_{k}(i)\right)=\left(\phi_{N+1}, \phi_{k}\right)=0 .
$$

By (5.7) and (5.11),

$$
\begin{aligned}
\left|\left(\phi_{N+1, i}-\phi_{N+1}, \psi_{k}(i)\right)\right| & \leq\left\|\phi_{N+1, i}-\phi_{N+1}\right\|_{2}\left\|\psi_{k}(i)\right\|_{2} \\
& \leq \frac{2 C \lambda_{k}^{n}}{i}
\end{aligned}
$$

From (C2), Proposition 2.1 and the hypothesis, it follows that for $k \leq N$

$$
\left\|\phi_{k}(i)\right\|_{\infty} \leq C \lambda_{k}(i)^{n} \leq C^{\prime}\left(\lambda_{k}(i)^{n}+1\right)
$$

where $C^{\prime}>0$ is a constant independent of $i$ and $k$. By (C1) and (5.24),

$$
\begin{aligned}
\left|\left(\phi_{N+1, i},\left(1-\rho_{i}\right) f_{i}^{*} \phi_{k}(i)\right)\right| & \leq\left\|\phi_{N+1, i}\right\|_{2}\left\|1-\rho_{i}\right\|_{2}\left\|\phi_{k}(i)\right\|_{\infty} \\
& \leq 2 C^{\prime}\left(\lambda_{k}^{n}+1\right)\left\|1-\rho_{i}\right\|_{2} \\
& \leq 2 C^{\prime}\left(\lambda_{k}^{n}+1\right) \gamma(i)
\end{aligned}
$$

In the same manner,

$$
\left|\left(\phi_{N+1, i}, f_{i}^{*} \phi_{k}(i)\left(G_{i}-1\right)\right)\right| \leq 2 C^{\prime}\left(\lambda_{k}^{n}+1\right)\left\|G_{i}-1\right\|_{2}
$$

Since $\left\|G_{i}-1\right\|_{\infty} \leq 2^{n}+1$ and $\lim _{i \rightarrow \infty}\left(G_{i}(x)-1\right)=0$ for almost every $x \in M$ by (C2) and (5.19), the Lebesgue convergence theorem implies

$$
\lim _{i \rightarrow \infty}\left\|G_{i}-1\right\|_{2}=0
$$

which combined with $(5.26)$ yields

$$
\lim _{i \rightarrow \infty}\left(\phi_{N+1, i}, f_{i}^{*} \phi_{k}(i)\left(G_{i}-1\right)\right)=0 .
$$

By (5.21), (5.22), (5.23), (5.35) and (5.28), we get

$$
\lim _{i \rightarrow \infty}\left(\chi_{N+1}(i), \phi_{k}(i)\right)_{i}=0,
$$

which yields

$$
\begin{aligned}
\lim _{i \rightarrow \infty}\left\|\chi_{N+1}(i)-\sum_{k \leq N}\left(\chi_{N+1}(i), \phi_{k}(i)\right)_{i} \phi_{k}(i)\right\|_{2, i}^{2} & =\lim _{i \rightarrow \infty}\left\|\chi_{N+1}(i)\right\|_{2, i}^{2} \\
& =\lim _{i \rightarrow \infty}\left\|\left(f_{i}^{-1}\right)^{*} \phi_{N+1}\right\|_{2, i}^{2} \\
& =1,
\end{aligned}
$$




$$
\begin{aligned}
\lim _{i \rightarrow \infty} \| d \chi_{N+1}(i) & -\sum_{k \leq N}\left(\chi_{N+1}(i), \phi_{k}(i)\right)_{i} d \phi_{k}(i) \|_{2, i}^{2} \\
& =\lim _{i \rightarrow \infty}\left\|d \chi_{N+1}(i)\right\|_{2, i}^{2} \\
& =\left\|d \phi_{N+1}\right\|_{2}^{2}+\lim _{i \rightarrow \infty} \int_{M-S_{\imath}}\left|d \phi_{N+1}\right|^{2}\left(G_{i}^{2}-1\right) d v
\end{aligned}
$$

By the Lebesgue convergence theorem again, we get

$$
\lim _{i \rightarrow \infty} \int_{M-S_{\imath}}\left|d \phi_{N+1}\right|^{2}\left(G_{i}^{2}-1\right) d v=0
$$

which combined with (5.30) and (5.31) yields

$$
\lim _{i \rightarrow \infty}\left\|d P\left(\phi_{N+1, i}\right)\right\|_{2, i}=\lambda_{N+1} .
$$

By (5.16) and (5.33) we get

$$
\lim _{i \rightarrow \infty} \sup \lambda_{N+1}(i) \leq \lambda_{N+1}
$$

Proof of Proposition 5.2. We prove the proposition by induction. It is clear by Theorem 3.1 that the proposition holds for $N=0$. Therefore we may assume the proposition for $0 \leq i \leq N$, and prove it for $N+1$.

By Lemma 5.2, we may assume $0<\lambda_{N+1}(i) \leq 2 \lambda_{N+1}$. By the definition, Proposition 2.1, (C1) and (C2), we have

$$
\begin{aligned}
\left\|\psi_{N+1}(i)\right\|_{2} & +\left\|d \psi_{N+1}(i)\right\|_{2} \\
& =\left\|\rho_{i} f_{i}^{*} \phi_{N+1}(i)\right\|_{2}+\left\|d\left(\rho_{i} f_{i}^{*} \phi_{N+1}(i)\right)\right\|_{2} \\
& \leq\left\|\rho_{i}\right\|_{2}\left\|\phi_{N+1}(i)\right\|_{\infty}+\left\|d \rho_{i}\right\|_{2}\left\|\phi_{N+1}(i)\right\|_{\infty} \\
& +\left\|\rho_{i}\right\|_{\infty}\left\|f_{i}^{*} d \phi_{N+1}(i)\right\|_{2} \\
& \leq 2 C \lambda_{N+1}^{n}+2 C \lambda_{N+1}^{n} \gamma(i)+2 C \lambda_{N+1}
\end{aligned}
$$

which combined with Proposition 5.1 implies that there is a subsequence $\{i(\nu)\}$ and $\psi \in W^{1,2}(M)$ such that

$$
\lim _{\nu \rightarrow \infty} \lambda_{N+1}(i(\nu))=\lim \inf _{i \rightarrow \infty} \lambda_{N+1}(i)
$$


and $\left\{\psi_{N+1}(i(\nu))\right\}$ converges to $\psi$ weakly in $W^{1,2}(M)$ and strongly in $L^{2}(M)$. Since

$$
\begin{aligned}
\left\|\psi_{N+1}(i)\right\|_{2} & =\left\|\rho_{i} f_{i}^{*} \phi_{N+1}(i)\right\|_{2} \\
& \geq\left\|f_{i}^{*} \phi_{N+1}(i)\right\|_{2}-\left\|\left(1-\rho_{i}\right) f_{i}^{*} \phi_{N+1}(i)\right\|_{2} \\
& \geq \frac{1}{2^{n}}\left\|\phi_{N+1}(i)\right\|_{L^{2}\left(M_{2}-K_{i}\right)}-C \lambda_{N+1}^{n}\left\|1-\rho_{i}\right\|_{2} \\
& \geq \frac{1}{2^{n}}\left(1-\left\|\phi_{N+1}(i)\right\|_{L^{2}\left(K_{\imath}\right)}\right)-C \lambda_{N+1}^{n} \gamma(i) \\
& \geq \frac{1}{2^{n}}\left(1-2 C \lambda_{N+1}^{n} \operatorname{vol}\left(K_{i}\right)\right)-C \lambda_{N+1}^{n} \gamma(i)
\end{aligned}
$$

we have

$$
\|\psi\|_{2}=\lim _{\nu \rightarrow \infty}\left\|\psi_{N+1}\left(i_{\nu}\right)\right\|_{2} \geq \frac{1}{2^{n}}
$$

which shows $\psi \neq 0$. Let us show that $\left(\psi, \phi_{k}\right)=0$ for $k \leq N$ and $\|\psi\|_{2}=1$. By computation,

$$
\begin{aligned}
& \begin{array}{l}
\left|\left(\psi_{N+1}(i), \psi_{k}(i)\right)\right| \\
\leq\left|\left(\rho_{i} f_{i}^{*} \phi_{N+1}(i), \rho_{i} f_{i}^{*} \phi_{k}\right)\right| \\
\leq|| 1-\rho_{i}^{2}\left\|_{2}\right\| \phi_{N+1}(i)\left\|_{\infty}\right\| \phi_{k}(i) \|_{\infty}+\left|\left(f_{i}^{*} \phi_{N+1}(i), f_{i}^{*} \phi_{k}(i)\right)\right| \\
\leq C \lambda_{N+1}^{2 n} \gamma(i)+\left|\int_{M_{2}-K_{i}} \phi_{N+1}(i) \phi_{k}(i) G_{i}^{-1} d v_{i}\right|
\end{array} \\
& \leq C \lambda_{N+1}^{2 n} \gamma(i)+\left|\left(\phi_{N+1}(i), \phi_{k}(i)\right)_{i}\right| \\
& \quad+\int_{M_{\imath}}\left|\phi_{N+1}(i) \phi_{k}(i)\left(G_{i}^{-1}-1\right) d v_{i}\right| \\
& \quad+\int_{K_{i}}\left|\phi_{N+1}(i) \phi_{k}(i) G_{i}^{-1} d v_{i}\right| \\
& \quad \leq C \lambda_{N+1}^{2 n} \gamma(i)+C 2^{n} \lambda_{N+1}^{2 n} \operatorname{vol}\left(K_{i}\right)+C \lambda_{N+1}^{2 n} \int_{M_{2}}\left|G_{i}^{-1}-1\right| d v_{i} \\
& \leq C \lambda_{N+1}^{2 n}\left(\gamma(i)+2^{n} \operatorname{vol}\left(K_{i}\right)+\int_{M}\left|G_{i}^{-1}-1\right| d v\right)
\end{aligned}
$$

where $G_{i}^{-1} \in L^{\infty}\left(M_{i}\right)$ is defined by

$$
G_{i}^{-1}:= \begin{cases}\left(d v_{i}-\left(f_{i}^{-1}\right)^{*} d v\right) / d v_{i} & \left(M_{i}-K_{i}\right) \\ 0 & \left(K_{i}\right)\end{cases}
$$


It is clear, by the definition, that $\left|G_{i}^{-1}\right|=\left|\left(f_{i}^{-1}\right)^{*} G_{i}\right| \leq 2^{n}$. By (C2) and the Lebesgue convergence theorem, we get

$$
\left(\psi, \phi_{k}\right)=\lim _{\nu \rightarrow \infty}\left(\psi_{N+1}(i(\nu)), \psi_{k}(i(\nu))=0\right.
$$

In the same way, we can show

$$
\|\psi\|_{2}=1
$$

Next let us show that $\Delta \psi=a \psi$ where $a=\liminf _{\nu \rightarrow \infty} \lambda_{N+1}(i(\nu))$.

Choose an arbitrary $\chi \in C_{0}^{\infty}(M)$. Since $\Delta=\delta_{\max } d_{\min }$ for $(M, g)$, it is sufficient to show

$$
(d \psi, d \chi)=a(\psi, \chi)
$$

to prove

$$
\Delta \psi=a \psi
$$

By the definition, we get

$$
\begin{aligned}
(d \psi, d \chi) & =\lim _{\nu \rightarrow \infty}\left(d \psi_{N+1}(i(\nu)), d \chi\right) \\
& =\lim _{\nu \rightarrow \infty}\left(d \phi_{N+1}(i(\nu)), d\left(\left(f_{i(\nu)}^{-1}\right)^{*} \chi\right)\right)_{i(\nu)} \\
& =\lim _{\nu \rightarrow \infty}\left(\Delta_{i(\nu)} \phi_{N+1}(i(\nu)),\left(f_{i(\nu)}^{-1}\right)^{*} \chi\right)_{i(\nu)} \\
& =\lim _{\nu \rightarrow \infty} \lambda_{N+1}(i(\nu))\left(\phi_{N+1}(i(\nu)),\left(f_{i(\nu)}^{-1}\right)^{*} \chi\right)_{i(\nu)} \\
& =a(\psi, \chi)
\end{aligned}
$$

which proves (5.43). Since $\psi \perp\left\{\phi_{0}, \cdots, \phi_{N}\right\}$ by $(5.40)$, we get

$$
a \geq \lambda_{N+1}=\inf _{f \perp\left\{\phi_{0}, \cdots, \phi_{N}\right\}}\|d f\|^{2} /\|f\|^{2} .
$$

Lemma 5.2 and (5.45) imply $\lambda_{N+1}=\lim \sup \lambda_{N+1}(i)=\liminf \lambda_{N+1}(i)$. This prove the proposition for $\mathrm{N}+1$.

Proof of Theorem 5.1. We prove the theorem by induction. Since $\lambda_{0}(i)$

0 for all $i$, it holds for $k=0$. We assume the theorem for $k \leq n$ and prove it for $k=n+1$. For the proof, it is sufficient to show

$$
\lim \sup _{i \rightarrow \infty} \lambda_{n+1}(i) \leq \lambda_{n+1} \leq \lim \inf _{i \rightarrow \infty} \lambda_{n+1}(i)
$$


We can choose sequences $\left\{i_{\nu}\right\}$ and $\left\{i_{\mu}\right\}$ such that

$$
\lim _{\nu \rightarrow \infty} \lambda_{n+1}\left(i_{\nu}\right)=\lim \sup _{i \rightarrow \infty} \lambda_{n+1}(i), \quad \lim _{\mu \rightarrow \infty} \lambda_{n+1}\left(i_{\mu}\right)=\lim \inf _{i \rightarrow \infty} \lambda_{n+1}(i) .
$$

By Propostioin 5.2, choosing subsequence $\left\{i_{\nu, k}\right\}$ and $\left\{i_{\mu, l}\right\}$ if needed, we have

$$
\lim _{k \rightarrow \infty} \lambda_{n+1}\left(i_{\nu, k}\right)=\lambda_{n+1}, \quad \lim _{l \rightarrow \infty} \lambda_{n+1}\left(i_{\mu, l}\right)=\lambda_{n+1} .
$$

This implies (5.46) and completes the proof.

\section{$\S 6$. Proof of Main Theorem}

In this section, we use the same notations as in $\S 0, \S 1, \S 3$ and $\S 5$.

In view of the proof of Theorem 5.1, it is sufficient to show the following proposition (P) to prove Main Theorem:

(P) For every sequence $\left\{t_{n}\right\}$ with $\lim _{n \rightarrow \infty} t_{n}=0$, there exists a subsequence $\left\{t_{n, i}\right\}$ such that

$$
\lim _{\nu \rightarrow \infty} \sigma\left(\Delta_{X_{t_{n, i}}}\right)=\sigma\left(\Delta_{Z}\right)
$$

Let $\left\{t_{n}\right\}$ be given. For every $i$, we can find $t_{n, i}$ with $\left|t_{n, i}\right|<\gamma(1 / i)$ where $\gamma(\epsilon)$ is the same one as in Theorem 1.1, and obtain a subsequence $\left\{t_{n, i}\right\}$. Set $(M, g):=\left(Z-\Sigma_{Z}, g_{Z}\right), M_{i}:=X_{t_{n, 2}}$ and $g_{i}:=g_{t_{n, 2}}$.

It is clear that $(M, g)$ is a Riemannian manifold with finite volume. To apply Theorem 5.1 to $(M, g)$ and $\left(M_{i}, g_{i}\right)$, we shall verify $(\mathrm{C} 1)$ and $(\mathrm{C} 2)$ for them.

At first we must find a family of cut-off functions verifying (C1) for $\left(Z-\Sigma_{Z}, g_{Z}\right)$. By Theorem 3.2 and Lemma 3.5, there is a family of cut-off functions $\left\{\rho_{i}\right\} \subset C_{0}^{0}\left(Z-\Sigma_{Z}\right) \cap W^{1,2}(Z)$ which satisfies $(\mathrm{C} 1)$ for $(Z, g)$ where $g:=\iota^{*}\left(\left.g_{\mathbb{P}^{N}(\mathbb{C})}\right|_{X_{0}}\right)$ is the pull back of the Bergmann metric of $X_{0}$. Since $g_{Z}$ is a restriction of some Riemannian metric of $\mathfrak{X}$, it is quasi-isometric to $g$. Therefore, $\left\{\rho_{i}\right\}$ also satisfies $(\mathrm{C} 1)$ for $\left(Z-\Sigma_{Z}, g_{Z}\right)$.

Next we must construct maps $f_{i}: M-S_{i} \rightarrow M_{i}$ verifying (C2) subject to $(\mathrm{C} 1)$. Set $f_{i}:=f_{t_{n, i}}^{(1 / i)}: Z-S_{i} \rightarrow X_{t_{n, i}}$ where $f_{t}^{\epsilon}: Z-\Sigma_{\epsilon} \rightarrow X_{t}$ is the same map constructed in Theorem 1.1. We remark that $f_{i}$ is welldefined on $Z-S_{i}$, since $f_{t_{n, i}}^{(1 / i)}$ is defined on $Z-\Sigma_{Z, \frac{1}{2}}$ by Theorem 1.1 and $Z-S_{i} \Subset Z-\Sigma_{Z, \frac{2}{2}}$ from Theorem 3.2. By Theorem 1.1 and (4.4), $\left\{f_{i}\right\}$ satisfies (C2) subject to (C1). 
Finally, we must verify the uniformity of the Sobolev inequality for $\left\{\left(M_{i}, g_{i}\right)\right\}$. But this follows from Corollary 4.2. Therefore, we can apply Theorem 5.1 to $\left(Z, g_{Z}\right)$ and $\left\{\left(X_{t_{n, i}}, g_{t_{n, i}}\right)\right\}$, and obtain (6.1).

\section{Appendix}

Let $X$ be an irreducible algebraic variety of dimension $n$ in $\mathbb{P}^{N}(\mathbb{C})$, and $Y$ an irreducible subvariety of dimension $d(<n)$ in $X$. For $y \in Y_{\text {reg }}$, let $N_{y}$ be a linear subspace of dimension $N-d$ in $\mathbb{P}^{N}(\mathbb{C})$ which contains $y$ such that $Y$ and $N_{y}$ intersects transversally at $y$; i.e., $T_{y} \mathbb{P}^{N}(\mathbb{C})=T_{y} Y \oplus T_{y} N_{y}$.

Here, by a linear subspace, we mean a subvariety of the form $H_{1} \cap \cdots \cap$ $H_{r}$ where $H_{i}$ is a hyperplane in $\mathbb{P}^{N}(\mathbb{C})$.

Proposition A.1. There exists a nonempty Zariski open subset $U_{Y}$ $\left(\subset Y-\Sigma_{Y}\right)$ of $Y$ such that if $y \in U_{Y}$, then

$$
\operatorname{dim}_{y} N_{y} \cap X=n-d
$$

For the proof, we need several lemmas. In what follows, rings in consideration are noetherian commutative with 1 , and all schemes are assumed to be Noetherian.

Let $A$ be a local ring and $\mathfrak{M}$ be the maximal ideal of $A$. Let $I$ and $J$ be proper ideals of $A$. Let $\bar{A}:=A / I$ be the residue ring and $\pi: A \rightarrow \bar{A}$ be the natural projection. Let $\overline{\mathfrak{M}}:=\pi(\mathfrak{M})$ be the maximal ideal of $\bar{A}$. We denote by $\operatorname{Gr}_{I} A$ the graded $\bar{A}$-algebra defined by

$$
\operatorname{Gr}_{I} A:=\oplus_{i=0}^{\infty} I^{i} / I^{i+1} \quad\left(I^{0}:=A\right) .
$$

For $x \in A$, we attach an integer $\nu_{I}(x)$ defined by

$$
\nu_{I}(x):=\sup \left\{j \in \mathbb{Z}_{\geq 0} ; x \in I^{j}\right\} \quad(x \neq 0), \quad \nu_{I}(0)=\infty
$$

For $x \in A$, we attach an element $\operatorname{in}_{I}(x)$ of $\operatorname{Gr}_{I} A$ defined by

$$
\operatorname{in}_{I}(x):=x \quad \bmod \quad I^{\nu_{I}(x)+1} \in I^{\nu_{I}(x)} / I^{\nu_{I}(x)+1} \quad(x \neq 0), \quad \operatorname{in}_{I}(0)=0 .
$$

Finally, we define an ideal $\operatorname{Gr}_{I}(J, A)$ of $\operatorname{Gr}_{I} A$ by

$$
\operatorname{Gr}_{I}(J, A):=\left\{\operatorname{in}_{I}(x) ; x \in J\right\}
$$


Lemma A.1. ([M, Theorem 15.7]) Let $A$ be a local ring, and $I \subsetneq A$ be an ideal. Then,

$$
\operatorname{dim} A=\operatorname{dim} \operatorname{Gr}_{I} A .
$$

LemmA A.2. ([H, Chap. 2, §2, Lemma 5]) There exists an isomorphism between graded algebras as follows:

$$
\operatorname{Gr}_{I+J / J} A / J \cong \operatorname{Gr}_{I} A / \operatorname{Gr}_{I}(J, A) .
$$

Lemma A.3. ([M, Theorem 15.1]) Let $\phi: A \rightarrow B$ be a flat local homomorphism of local rings. Then,

$$
\operatorname{dim} B=\operatorname{dim} A+\operatorname{dim} B / \mathfrak{M} B
$$

Lemma A.4. Let $A$ be a local ring with the maximal ideal $\mathfrak{M}$, and $B$ be a flat $A$-algebra with $B \neq \mathfrak{M} B$. Then,

$$
\operatorname{dim} B \geq \operatorname{dim} A+\operatorname{dim} B / \mathfrak{M} B
$$

Proof. Take a maximal ideal $\overline{\mathfrak{n}}$ of $B / \mathfrak{M} B$ such that $\operatorname{dim} B / \mathfrak{M} B=$ $\operatorname{dim}(B / \mathfrak{M} B)_{\overline{\mathfrak{n}}}$ where $(B / \mathfrak{M} B)_{\overline{\mathfrak{n}}}$ is the localization of $B / \mathfrak{M} B$ by the maximal ideal $\overline{\mathfrak{n}}$.

Let $\pi: B \rightarrow B / \mathfrak{M} B$ be the projection. We set $\mathfrak{n}:=\pi^{-1}(\overline{\mathfrak{n}})$. Then, by Lemma A.3, we have

$$
\operatorname{dim} B \geq \operatorname{dim} B_{\mathfrak{n}}=\operatorname{dim} A+\operatorname{dim}(B / \mathfrak{M} B)_{\overline{\mathfrak{n}}}=\operatorname{dim} A+\operatorname{dim} B / \mathfrak{M} B
$$

Finally, we need the following theorem:

Theorem A.1. ([H, Chap. 2, Sect. 1, Theorem 1]) Let $X$ be a scheme and $Y \subset X$ be a reduced closed subscheme. Then, there exists a nonempty Zariski open subset $U \subset X$ such that

(1) $U \cap Y \neq \emptyset$.

(2) $U$ is normally flat along $U \cap Y$. Or equivalently, $\operatorname{Gr}_{\mathcal{I}_{Y, y}} \mathcal{O}_{X, y}$ is $\mathcal{O}_{Y, y^{-}}$ flat for every $y \in U \cap Y$ where $\mathcal{O}_{X}$ (resp. $\left.\mathcal{O}_{Y}\right)$ is the structure sheaf of $X$ (resp. $Y), \mathcal{I}_{Y}\left(\subset \mathcal{O}_{X}\right)$ is the defining ideal sheaf of $Y$, and $\mathcal{O}_{X, y}$ (resp. $\mathcal{O}_{Y, y}$, $\left.\mathcal{I}_{Y, y}\right)$ denotes the stalk at $y$. 
Proof of Proposition A.1. By virtue of Theorem A.1, it suffices to prove that $\operatorname{dim}_{y} N_{y} \cap X=n-d$ if $y \in Y_{\text {reg }}$ and if $X$ is normally flat along $Y$ at $y$; i.e., $\operatorname{Gr}_{\mathcal{I}_{Y, y}^{X}} \mathcal{O}_{X, y}$ is $\mathcal{O}_{Y, y}$-flat.

We can take a minimal set of generators $z=\left(z_{1}, \cdots, z_{d}\right)$ (consisting of $d$-elements) of $\mathcal{I}_{N_{y}, y}^{\mathbb{P}^{N}}$. Set $A=\mathcal{O}_{X, y}, \mathfrak{M}=\mathfrak{M}_{X, y}$ and $I=\mathcal{I}_{Y, y}$. We also set $\bar{A}=A / I$ and $\overline{\mathfrak{M}}=\mathfrak{M} / I$.

Since $\operatorname{dim}_{y} N_{y} \cap X \geq n-d$ from the intersection inequality of dimension (cf. [G-R, p. 102]), it suffices to show $\operatorname{dim}_{y} N_{y} \cap X \leq n-d$. By Lemma A.1 and A.2,

$$
\begin{aligned}
\operatorname{dim}_{y} N_{y} \cap X & =\operatorname{dim} A / z A \\
& =\operatorname{dim} \operatorname{Gr}_{(I+z A) / z A} A / z A=\operatorname{dim} \operatorname{Gr}_{I} A / \operatorname{Gr}_{I}(z A, A)
\end{aligned}
$$

As $Y$ and $N_{y}$ intersects transversally at $y$, we have $\mathcal{I}_{N_{y}, y}^{\mathbb{P}^{N}}+\mathcal{I}_{Y, y}^{\mathbb{P}^{N}}=\mathfrak{M}_{\mathbb{P}^{N}, y}$. Hence, $z$ is mapped to the regular system of parameter $\bar{z}=\left(\bar{z}_{1}, \cdots, \bar{z}_{d}\right)$ of $\bar{A}=A / I=\mathcal{O}_{Y, y}=\mathcal{O}_{\mathbb{P}^{N}, y} / \mathcal{I}_{Y, y}^{\mathbb{P}^{N}}$ by the natural projection map. In other words, we have $\bar{z} \bar{A}=\overline{\mathfrak{M}}$. This also shows that $\nu_{I}\left(z_{i}\right)=0$ for each $i$; i.e., $z_{i} \notin$ $I$ for each $i$. Here we consider $z_{i}$ as an element of $A=\mathcal{O}_{X, y}=\mathcal{O}_{\mathbb{P}^{N}, y} / \mathcal{I}_{X, y}^{\mathbb{P}^{N}}$. This shows that $\operatorname{in}_{I}\left(z_{i}\right)=z_{i} \bmod I=\bar{z}_{i}$ is an element of the degree zero component $\bar{A}=A / I$ of $\operatorname{Gr}_{I} A$.

As $\operatorname{Gr}_{I}(z A, A)$ contains $\bar{z}$, it suffices to show that $\operatorname{dim} \operatorname{Gr}_{I} A / \bar{z} \operatorname{Gr}_{I} A \leq$ $n-d$ by (A.5). Note that we have $\operatorname{Gr}_{I} A / \bar{z} \operatorname{Gr}_{I} A \cong \operatorname{Gr}_{I} A / \overline{\mathfrak{M}}_{\mathrm{Gr}_{I}} A$. Hence, by Lemma A.1 and A.4, we have

(A.6) $\operatorname{dim} \operatorname{Gr}_{I} A / \bar{z} \operatorname{Gr}_{I} A \leq \operatorname{dim} \operatorname{Gr}_{I} A-\operatorname{dim} A / I=\operatorname{dim} A-\operatorname{dim} A / I=n-d$.

This completes the proof.

Proposition A.2. Let $X$ be an irreducible algebraic variety of dimension $n$ in $\mathbb{P}^{N}(\mathbb{C})$.

Let $H_{1}, \cdots, H_{r} \quad(r \leq n)$ be hyperplanes in $\mathbb{P}^{N}(\mathbb{C})$. Let

$$
\begin{aligned}
& X \cap H_{1} \cap \cdots \cap H_{r}=\bigcup_{i=0}^{r}\left(X \cap H_{1} \cap \cdots \cap H_{r}\right)^{(n-i)} \\
& \left(\operatorname{dim}\left(X \cap H_{1} \cap \cdots \cap H_{r}\right)^{(n-i)}=n-i\right)
\end{aligned}
$$

be the decomposition into the pure dimensional components. Then,

$$
\operatorname{deg}(X) \geq \operatorname{deg}\left(X \cap H_{1} \cap \cdots \cap H_{r}\right)_{\text {prop }}
$$


where $\left(X \cap H_{1} \cap \cdots \cap H_{r}\right)_{\text {prop }}:=\left(X \cap H_{1} \cap \cdots \cap H_{r}\right)^{(n-r)}$ is the proper component of the intersection $X \cap H_{1} \cap \cdots \cap H_{r}$.

For the proof, we prepare a lemma.

LEMma A.5. Let $Y$ be an irreducible algebraic variety in $\mathbb{P}^{N}(\mathbb{C})$ and $H$ be a hyperplane in the same projective space. Then,

$$
\operatorname{deg}(Y) \geq \operatorname{deg}(Y \cap H)_{\text {prop }}
$$

Proof. By the intersection inequality of dimension (cf. [G-R, p. 102]), $\operatorname{dim} Y \cap H$ is equal to either $\operatorname{dim} Y-1$ or $\operatorname{dim} Y$. When $\operatorname{dim} Y \cap H=$ $\operatorname{dim} Y-1$, then $\operatorname{deg}(Y)=\operatorname{deg}(Y \cap H)$ by the Bézout theorem. When $\operatorname{dim} Y \cap H=\operatorname{dim} Y, Y \cap H=Y$ and therefore $(Y \cap H)_{\text {prop }}=\emptyset$. Therefore, $\operatorname{deg}(Y \cap H)_{\text {prop }}=0$ by the definition. This completes the proof.

Proof of Proposition A.2. We prove the propositon by induction. When $r=0$, there is nothing to prove. We assume the proposition for $r \leq k$ and prove it for $r=k+1$.

Let

$$
\left(X \cap H_{1} \cap \cdots \cap H_{r}\right)_{\text {prop }}=\bigcup_{\alpha} A_{\alpha}
$$

be the irreducible decomposition. By the definition and the intersection inequality of dimension,

$$
\left(X \cap H_{1} \cap \cdots \cap H_{r} \cap H_{k+1}\right)_{\text {prop }} \subset \bigcup_{\alpha}\left(A_{\alpha} \cap H_{k+1}\right)_{\text {prop }}
$$

By Lemma A.5 and the hypothesis of induction, we have

$$
\begin{aligned}
\operatorname{deg}(X) \geq \sum_{\alpha} \operatorname{deg}\left(A_{\alpha}\right) & \geq \sum_{\alpha} \operatorname{deg}\left(A_{\alpha} \cap H_{k+1}\right)_{\text {prop }} \\
& \geq \operatorname{deg}\left(X \cap H_{1} \cap \cdots \cap H_{k+1}\right)_{\text {prop }}
\end{aligned}
$$

This completes the proof. 


\section{REFERENCES}

[A] A. Akutagawa, Convergence for Yamabe metrics of positive scalar curvature with integral bounds on curvature, preprint (1994).

[B-K-N] S. Bando, A. Kasue and H. Nakajima, On a construction of coordinates at infinity on manifolds with fast curvature decay and maximal volume growth, Invent. Math., 97 (1989), 313-349.

[C-K-S] E. A. Carlen, S. Kusuoka and P. W. Stroock, Upper bounds for symmetric Markov transition functions, Ann. Inst. H. Poincaré, 23 (1987), 245-287.

[C] I. Chavel, Eigenvalues in Riemannian Geometry, Academic Press, New York, 1984.

[C-F] I. Chavel and E. A. Feldman, Spectra of domains in compact manifolds, J. Funct. Anal., 30 (1978), 198-222.

[C-L] S.-Y. Cheng and P. Li, Heat kernel estimates and lower bound of eigenvalues, Comment. Math. Helv., 56 (1981), 327-338.

[C-L-Y] S.-Y. Cheng, P. Li and S.-T. Yau, Heat equations on minimal submanifolds and their applications, Amer. J. Math., 106 (1984), 1033-1065.

[Cl] C. H. Clemens, Degeneration of Kähler manifolds, Duke Math. J., 44 (1977), 215-290.

[D] E. B. Davis, Heat kernels and spectral theory, Cambridge Univ. Press, Cambridge, 1989.

[F] A. Fujiki, Closedness of the Duady spaces of compact Kähler spaces, Publ. RIMS, Kyoto Univ., 14 (1978), 1-52.

[Fk] K. Fukaya, Collapsing Riemannian manifolds and eigenvalues of the Laplace operator, Invent. Math., 87 (1987), 517-547.

[G-H] P. Griffiths and J. Harris, Principles of algebraic geometry, John Wiley, New York, 1978.

[G-R] H. Grauert and R. Remmert, Coherent analytic sheaves, Springer, Berlin, Heidelberg, New York, 1984.

[G-T] D. Gilberg and N. S. Trudinger, Elliptic partial differential equations of second order (2nd ed.), Springer, Berlin, Heidelberg, New York, 1983.

[G] A. Gray, Tubes, Addison-Wesley, New York, 1990.

[Gr1] M. Gromov, Spectral geometry of semi-algebraic sets, Ann. Inst. Fourier, 42 (1992), 249-274.

[Gr2] — Metric invariants of Kähler manifolds, preprint (1992).

$[\mathrm{H}] \quad \mathrm{H}$. Hironaka, Resolution of singularities of an algebraic variety over a field of characteristic zero, Ann. of Math., 79 (1964), 109-326.

[J-W] L. Ji and R. Wentworth, Spectral convergence on degenerating surfaces, Duke Math. J., 66 (1992), 469-501.

[K-K1] A. Kasue and H. Kumura, Spectral convergence of Riemannian manifolds, Tôhoku Math. J., 46 (1994), 147-179.

[K-K2] _ Spectral convergence of Riemannian manifolds, II, Tohoku Math. J., 48 (1996), 71-120.

[L-T] P. Li and G. Tian, On the heat kernel of the Bergmann metric on algebraic 
varieties, J. Amer. Math. Soc., 8 (1995), 857-877.

[M] H. Matsumura, Commutative ring theory, Cambridige University Press, 1986.

[N] H. Nakajima, Hausdorff convergence of Einstein 4-manifolds, J. Fac. Sci. Univ. Tokyo, 35 (1988), 411-424.

[Na] S. Nakano, Several complex variables, (in Japanese), Asakura shoten Publ., Tokyo, 1981.

[Y1] K. Yoshikawa, Conic degeneration of Riemannian manifolds and the spectral zeta function, preprint (1993).

[Y2] Degeneration of complex manifolds and the spectrum of Laplace operator, (in Japanese), Master thesis at Kyoto University (1992).

Nagoya University

Chikusa-ku, Nagoya 464-01

Japan

yosikawa@math.nagoya-u.ac.jp 